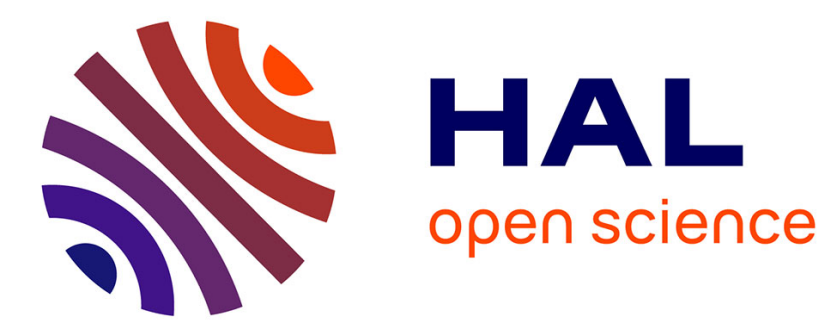

\title{
Ambiguity Aversion and Incompleteness of Financial Markets
}

Sujoy Mukerji, Jean-Marc Tallon

\section{To cite this version:}

Sujoy Mukerji, Jean-Marc Tallon. Ambiguity Aversion and Incompleteness of Financial Markets. Review of Economic Studies, 2001, pp.883-904. halshs-00174539

\section{HAL Id: halshs-00174539 \\ https://shs.hal.science/halshs-00174539}

Submitted on 24 Sep 2007

HAL is a multi-disciplinary open access archive for the deposit and dissemination of scientific research documents, whether they are published or not. The documents may come from teaching and research institutions in France or abroad, or from public or private research centers.
L'archive ouverte pluridisciplinaire HAL, est destinée au dépôt et à la diffusion de documents scientifiques de niveau recherche, publiés ou non, émanant des établissements d'enseignement et de recherche français ou étrangers, des laboratoires publics ou privés. 


\title{
Ambiguity Aversion and Incompleteness of Financial Markets ${ }^{1}$
}

\author{
Sujoy Mukerji² \& Jean-Marc Tallon ${ }^{3}$
}

December 15, 2000

${ }^{1}$ We thank the referees and the Managing Editor, M. Armstrong, as well as, A. Bisin, S. Bose, P. Ghirardato, I. Gilboa, R. Guesnerie, B. Lipman, J. Malcomson, J. Marin, M. Marinacci, M. Piccione, Z. Safra, H. S. Shin and J. C. Vergnaud for helpful comments. The paper has also benefitted from the responses of seminar members at the Univ. of British Columbia, Univ. of Essex, Univ. Evry, Johns Hopkins, Nuffield College, Tilburg Univ., CORE-Louvain-la-Neuve, NYU, UPenn, Univ. of Paris I, Univ. of Toulouse, Univ. du Maine, ENPC-Paris, Univ. Venezia and the ESRC Economic Theory Conference at Kenilworth. The first author gratefully acknolwedges financial assistance from an Economic and Social Research Council of U.K. Research Fellowship (\# R000 27 1065).

${ }^{2}$ Corresponding author. Department of Economics, University of Oxford, Manor Road Building, Manor Road, Oxford OX1 3UL, United Kingdom. e-mail:sujoy.mukerji@economics.ox.ac.uk

${ }^{3}$ EUREQua-CNRS, Université Paris I Panthéon-Sorbonne, 106-112 bld de l'Hôpital, 75647 Paris Cedex 13, France. e-mail:jmtallon@univ-paris1.fr 


\begin{abstract}
It is widely thought that incomes risks can be shared by trading in financial assets. But financial assets typically carry some risk idiosyncratic to them, hence, disposing incomes risk using financial assets will involve buying into the inherent idiosyncratic risk. However, standard theory argues that diversification would reduce the inconvenience of idiosyncratic risk to arbitrarily low levels. This paper shows that this argument is not robust: ambiguity aversion can exacerbate the tension between the two kinds of risks to the point that classes of agents may not want to trade some financial assets. Thus, theoretically, the effect of ambiguity aversion on financial markets is to make the risk sharing opportunities offered by financial markets less complete than it would be otherwise.
\end{abstract}

JEL Nos: D81, D52

Keywords: Ambiguity aversion, incomplete markets, sub-optimal risk sharing. 


\section{Introduction}

Suppose an agent's subjective knowledge about the likelihood of contingent events is consistent with more than one probability distribution. And further that, what the agent knows does not inform him of a precise (second order) probability distribution over the set of 'possible' (first order) probabilities. We say then that the agent's beliefs about contingent events are characterized by ambiguity. If ambiguous, the agent's beliefs are captured not by a unique probability distribution in the standard Bayesian fashion but instead by a set of probabilities. Thus not only is the outcome of an act uncertain but also the expected payoff of the action, since the payoff may be measured with respect to more than one probability. An ambiguity averse decision maker evaluates an act by the minimum expected value that may be associated with it: the decision rule is to compute all possible expected values for each action and then choose the act which has the best minimum expected outcome. This (informal) notion of ambiguity aversion inspires the formal model of Choquet expected utility (CEU) preferences introduced in Schmeidler (1989). The present paper considers a model of financial markets populated by agents with CEU preferences, with the interpretation that the agents' preferences demonstrate ambiguity aversion ${ }^{1}$.

Typically, economic agents are endowed with income streams that are not evenly spread over time or across uncertain states of nature. A financial contract is a claim to a contingent income stream-hence the logic of the financial markets: by exchanging such claims agents change the shapes of their income streams, obtaining a more even consumption across time and the uncertain contingencies. A financial market is said to be complete if contingent payoffs from the different marketed financial contracts are varied enough to span all the contingencies. However, casual empiricism suggests that in just about every financial market in the real world the span is less than the full set of contingencies, i.e., the markets are incomplete. The primary implication of incompleteness of financial markets is that agents may transfer income only across a limited set of contingencies and are thus left to share risk in a suboptimal manner ${ }^{2}$.

Consider the following question: Take a (financial) economy with complete markets, but suppose agents are not subjective expected utility (SEU) maximizers, but rather CEU maximizers; are there conditions under which it is possible that at a competitive equilibrium agents do not trade some assets and hence their equilibrium allocations are equivalent to competitive allocations deriving from some incomplete market economy wherein the allocations are not Pareto optimal? The answer to the question is a qualified yes. The qualification is important and the essential contribution of the present paper is in identifying this qualification. Imposing CEU maximization in a complete market economy does not generate no-trade, but, as this paper shows, a robust sequence of incomplete market economies which would converge to complete markets with SEU agents but does not with CEU, can be constructed. The key characteristic of such a sequence of economies is that they include, as non-redundant instruments of risk-sharing, financial assets which are affected by idiosyncratic risk ${ }^{3}$. We establish that trade in financial assets, whose payoffs have idiosyncratic components, may break down because of ambiguity

\footnotetext{
${ }^{1}$ Recent literature has debated the merits of the CEU framework as a model of ambiguity aversion. For instance, Epstein (1999) contends that CEU preferences associated with convex capacities (see section 2, below) do not always conform with a "natural" notion of ambiguity averse behavior. On the other hand, Ghirardato and Marinacci (1997) argue that ambiguity aversion is demonstrated in the CEU model by a broad class of capacities which includes convex capacities.

${ }^{2}$ And, indeed, formal empirical investigations overwhelmingly confirm that the data on individual consumption are more consistent with incomplete than complete markets. Among others, see Zeldes (1989), Carroll (1992), Deaton and Paxson (1994) and Hayashi, Altonji, and Kotlikof (1996). The evidence, however, is not unanimous, see e.g., Mace (1991).

${ }^{3}$ We will say an asset's payoff has an idiosyncratic component if at least some component of the payoff is independent of (1) the realized endowments of agents and (2) of the payoff of any other asset as well.
} 
aversion. We find, furthermore, that the no-trade due to ambiguity aversion is a robust occurrence, in the sense that it takes place even in the limit replica economy, with enough replicas of the financial assets such that idiosyncratic risk may be completely hedged. Hence, the behavior of the limit replica economy is markedly different depending on whether agents are SEU maximizers or CEU maximizers: in the former case the allocation is precisely that of a complete markets economy whereas in the latter case, because of the endogenous breakdown of trade, the equilibrium allocation, given a "high enough" level of ambiguity aversion and idiosyncratic risk, is not Pareto optimal and the nature of risk-sharing is as in an incomplete markets economy. These findings are of interest, both for the way it complements the related literature and for the substantive economic insight it gives rise to.

Dow and Werlang (1992) showed, in a model with one risky and one riskless asset, a single ambiguity averse agent with CEU preferences, exogenously determined asset prices, and a riskless initial endowment, that there exists a non-degenerate price interval at which an agent will strictly prefer to take a zero position in the risky asset. Recall, the logic of this result essentially rests on the observation that a CEU agent when going short in the risky asset will use a different probability to evaluate expected return as compared to when going long, since an agent taking a short (long) position is relatively better (respectively, worse) off in states where the asset payoff is shocked adversely. Having (robustly) rationalized a zero position in a single decision-maker framework one might be tempted to conjecture (even though such a conjecture is not made by Dow and Werlang) that it were but a short step to generate no-trade in a full equilibrium model. But, as we remarked above, simply imposing CEU maximization in a complete market economy does not generate no-trade unless endowments are Pareto optimal to begin with. The point is that, with complete markets, allocations are Pareto optimal and hence comonotonic (i.e., every agent's ranking of states, ranked in accordance with the agent's ex post utility from the given allocation, is identical) (Chateauneuf, Dana, and Tallon (2000)). Comonotonicity implies that all agents evaluate the returns of assets with the same probability measure in a CEU world. Thus, closing Dow and Werlang model in the obvious way makes it apparent that, for generic endowments, assets will surely be traded. Hence it is, at least, of academic interest to find what condition actually generates an endogenous closure of some financial markets and a consequent lessening of risk-sharing opportunities, when moving from an SEU to a CEU world.

Perhaps, a more compelling reason for interest in our findings is their economic significance. It is widely regarded that a crucial function of financial markets is that they allow individuals to hedge their income (from, say, human capital/labor) risk even though such risks are not, per se, contractible in appropriate detail because of usual reasons of asymmetric information and/or transactions costs. For instance, take X, a shopowner in Detroit, whose fortunes are heavily dependent on the fortunes of the automobile industry centered in Detroit. While $\mathrm{X}$ would love to smooth consumption across the various possible income shocks, it is hardly likely that an insurance company would be willing to insure $\mathrm{X}$ against anything other than accidents like fire and theft. But, standard economic/finance theory would argue, even though such personalized contracts may not be available, X should be able to hedge his income shocks in the stock market. To transfer income from the "good" states to the "bad", all that is required is that X take a short position on a portfolio of shares of different firms in automobile (and related) industry and a long position on a "safe" asset (e.g., a government bond). Of course, the returns of any particular share will not be perfectly correlated with X's income; in particular, each individual share return will be subject to some idiosyncratic risk. But, with a large enough number of such equities in the portfolio, the idiosyncracies may be hedged away, and X would find the (almost) perfect hedge for his income shocks. To X, therefore, for all practical purposes, the economy is very much a complete markets economy.

However, what this paper shows is that the story only runs so far in an SEU world, not in 
a CEU world. Consider two agents trading an equity subject to idiosyncratic risk, with one agent taking a short position while the other goes long. Evidently, then, the variation in the agents' consumption across states which differ only in terms of the idiosyncratic shocks would be exclusively determined by the nature and extent of the shocks and the agents' position on the asset. Moreover, the variation of each agent's consumption across such states will be inversely related, and therefore, their consumption will not be comonotonic. Hence, given ambiguity aversion with CEU preferences, an agent will behave as if he applies a different probability measure depending on whether he is choosing to go short or to go long. Therefore, it may be that the minimum asking price of the agent when choosing to go short will be higher than the maximum bid of the agent when choosing to go long. Thus, no trade may result, and the paper provides sufficient conditions that obtain the result. Indeed, as we show, the no trade outcome will survive even in the limit, when there are an arbitrarily large number of (independent) replicas of the equity. The intuition here is that the law of large numbers implies that the agents' beliefs on the payoff of a portfolio of risky assets, hit (in part) by idiosyncratic shocks, converge to some mean, but the mean is in principle different for agents taking differently signed positions on the (relevant) assets. In this fashion, ambiguity aversion creates an endogenous limit to the extent of risk sharing possible through financial markets, thereby providing a (theoretical) justification for the basic premise of the general equilibrium with incomplete markets model (GEI). To see it in the eyes of X, in a CEU world, unlike in an SEU world, there may not exist prices that would allow $\mathrm{X}$ to go short on automobile industry equities as he needs to do to "export" his income risk. The same market which offers possibilities of risk sharing equivalent to complete markets when beliefs and behavior are in accordance with SEU, offers only the Pareto sub-optimal risk sharing possibilities of an incomplete market economy when agents are CEU maximizers with beliefs that are "sufficiently" ambiguous.

The rest of the paper is organized as follows. The next section provides an introduction to the formal model of ambiguity aversion applied in this paper. Section 3 contains the formal model of the finance economy and the main result. Section 4 concludes the paper. Appendix A contains some technical material on independence and law of large numbers for capacities. All formal proofs are in the Appendix B.

\section{Choquet expected utility and the related literature}

Let $\Omega=\left\{\omega_{i}\right\}_{i=1}^{N}$ be a finite state space, and assume that the decision maker (DM) chooses among acts with state contingent payoffs, $z: \Omega \rightarrow \mathbb{R}$. In the CEU model ( Schmeidler (1989)) an ambiguity averse DM's subjective belief is represented by a convex non-additive probability function (or a convex capacity), $\nu$ such that, (i) $\nu(\emptyset)=0$, (ii) $\nu(\Omega)=1$ and, (iii) $\nu(X \cup Y) \geq$ $\nu(X)+\nu(Y)-\nu(X \cap Y)$, for all $X, Y \subset \Omega$. Define the core of $\nu$, (notation: $\Delta(\Omega)$ is the set of all additive probability measures on $\Omega$ ):

$$
\mathcal{C}(\nu)=\{\mu \in \Delta(\Omega) \mid \mu(X) \geq \nu(X), \text { for all } X \subset \Omega .\}
$$

Hence, $\nu(X)=\min _{\mu \in \mathcal{C}(\nu)} \mu(X)$. The ambiguity ${ }^{4}$ of the belief about an event $X$ is measured by the expression $\mathcal{A}(X ; \nu) \equiv 1-\nu(X)-\nu\left(X^{c}\right)=\max _{\mu \in \mathcal{C}(\nu)} \mu(X)-\min _{\mu \in \mathcal{C}(\nu)} \mu(X)$.

Like in SEU, a utility function $u: \mathbb{R}_{+} \rightarrow \mathbb{R}, u^{\prime}(\cdot) \geq 0$, describes DM's attitude to risk and wealth. Given a convex non-additive probability $\nu$, the Choquet expected utility ${ }^{5}$ of an act is

\footnotetext{
${ }^{4}$ Fishburn (1993) provides an axiomatic justification of this definition of ambiguity and Mukerji (1997) demonstrates its equivalence to a more primitive and epistemic notion of ambiguity (expressed in term's of the DM's knowledge of the state space).

${ }^{5}$ The Choquet expectation operator may be directly defined with respect to a non-additive probability, see Schmeidler (1989). Also, for an intuitive introduction to the CEU model see Section 2 in Mukerji (1998).
} 
simply the minimum of all possible 'standard' expected utility values obtained by measuring the contingent utilities possible from the act with respect to each of the additive probabilities in the core of $\nu$ :

$$
\mathbb{C E}_{\nu} u(z)=\min _{\mu \in \mathcal{C}(\nu)}\left\{\sum_{\omega \in \Omega} u(z(\omega)) \mu(\omega)\right\}
$$

The fact that the same additive probability in $\mathcal{C}(\nu)$ will not in general 'minimize' the expectation for two different acts, explains why the Choquet expectations operator is not additive, i.e., given any acts $z, w: \mathbb{C E}_{\nu}(z)+\mathbb{C E}_{\nu}(w) \leq \mathbb{C E}_{\nu}(z+w)$. The operator is additive, however, if the two acts $z$ and $w$ are comonotonic, i.e., if $\left(z\left(\omega_{i}\right)-z\left(\omega_{j}\right)\right)\left(w\left(\omega_{i}\right)-w\left(\omega_{j}\right)\right) \geq 0$.

In our analysis, we will need to consider the independent product of capacities. The independent product of two convex capacities $\nu_{1}$ and $\nu_{2}$, according to the definition (suggested by Gilboa and Schmeidler (1989)) we apply in this paper, may be (informally) understood as the lower envelope of the set $\left\{\mu_{1} \times \mu_{2} \mid \mu_{1} \in \mathcal{C}\left(\nu_{1}\right), \mu_{2} \in \mathcal{C}\left(\nu_{2}\right)\right\}$. Unlike what is true with "standard" probabilities, there is more than one way to define the independent product of two capacities. As it turns out, the formal analysis in this paper is unaffected if an alternative definition of independence were applied. We refer the interested reader to the Appendix A and to the discussion at the end of Section 3 for more on the independent product of capacities and turn next to the use of capacities and CEU in portfolio decision problems.

Dow and Werlang (1992), as noted earlier, identified an important implication of Schmeidler's model They showed, in a model with one risky and one riskless asset, that if a CEU maximizer has a riskless endowment then there exists a set of asset prices that support the optimal choice of a riskless portfolio. The intuition behind this finding may be grasped in the following example. Consider an asset that pays off 1 in state $L$ and 3 in state $H$ and assume that $\nu(L)=0.3$ and $\nu(H)=0.4$. Assuming that the DM has a linear utility function, the expected payoff of buying an unit of $z$, the act $z_{b}$, is given by $\mathbb{C E}_{\nu}\left(z_{b}\right)=0.6 \times 1+0.4 \times 3=1.8$. On the other hand, the payoff from going short on an unit of $z$ (the act $z_{s}$ ) is higher at $L$ than at $H$. Hence, the relevant minimizing probability when evaluating $\mathbb{C E}_{\nu}\left(z_{b}\right)$ is that probability in $\mathcal{C}(\nu)$ that puts most weight on $H$. Thus, $\mathbb{C E}_{\nu}\left(z_{s}\right)=0.3 \times(-1)+0.7 \times(-3)=-2.4$. Hence, if the price of the asset $z$ were to lie in the open interval $(1.8,2.4)$, then the investor would strictly prefer a zero position to either going short or buying. Unlike in the case of unambiguous beliefs there is no single price at which to switch from buying to selling. Taking a zero position on the risky asset has the unique advantage that its evaluation is not affected by ambiguity.

The 'inertia' zone demonstrated by Dow and Werlang was simply a statement about optimal portfolio choice corresponding to exogenously determined prices, given an initially riskless position. However, it does not follow from this result at the individual level that no-trade is an equilibrium when closing the model by allowing agents to trade their risks, as we illustrate next using the Edgeworth box diagram in Figure 1.

The diagram depicts the possibilities of risk-sharing (one may think of the risk sharing as being obtained through the exchange of two Arrow securities, one for each contingency) between two CEU agents, $h=1,2$, with uncertain endowment in the two states $\omega_{a}$ and $\omega_{b}$. W is the endowment vector. Notice that, because of ambiguity aversion, the indifference curves are kinked at the point of intersection with the $45^{\circ}$ ray through the origin. The shaded area in the diagram represents the area of mutually advantageous trade. Hence, no-trade is an equilibrium outcome in this economy if and only if endowment is Pareto optimal to begin with. Introduction of ambiguity aversion in an economy, seemingly, would not impede the trade in risk sharing contracts and would not be a reason for incomplete risk sharing. The reason for this "absence of no-trade" goes as follows: Pareto optimal allocations lie within the "tramlines', the $45^{0}$ rays through each origin, i.e., they are comonotonic. Hence, at a Pareto optimal 


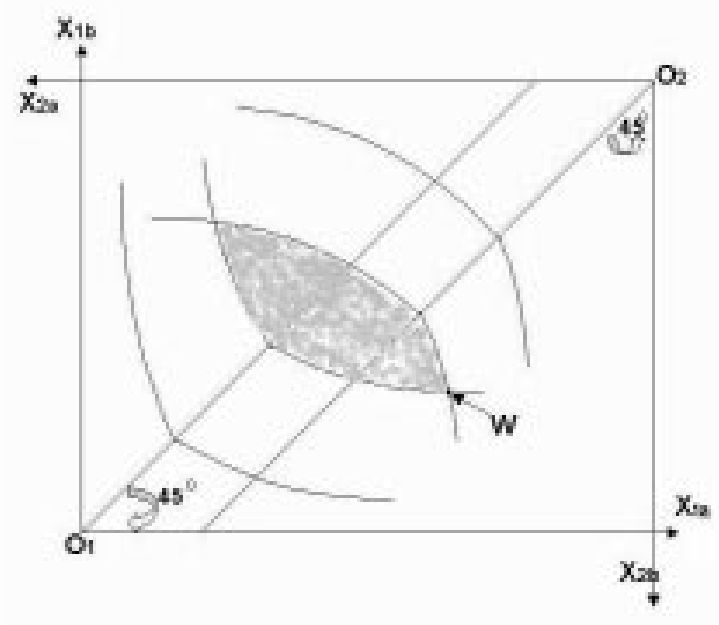

Figure 1:

allocation, the ranking of the states is identical for both agents and is given by the ordering of aggregate endowment. Now with complete markets, equilibrium allocations are Pareto optimal and therefore comonotonic as well. Thus, agents use the same 'minimizing probability' at equilibrium, and agree on asset valuation. Risk sharing proceeds just as in an economy with SEU agents (see Chateauneuf, Dana, and Tallon (2000)).

Thus, if one wants to obtain that equilibrium be characterized by absence of trade, one has to move away from this (canonical) example, something that is accomplished by introducing into the model assets with idiosyncratic payoff components. Epstein and Wang (1994) recognized the role of first of the two conditions defining idiosyncratic risk (as defined in this paper) in obtaining non-unique equilibrium asset prices in a CEU world. That result is related to ours. The precise relationship between the results deserves careful discussion. For expository purposes, we turn to this discussion at the end of the next section, after the presentation of our model.

We end this section with a discussion of another model of behavior under Knightian uncertainty due to Bewley (1986), distinct from the one applied in this paper, which easily generates a no-trade result. Bewley, essentially, drops Savage's assumption that preferences are complete and adds an axiom of the 'status quo'. In our Edgeworth box this would amount to assuming that indifference curves are kinked precisely at the endowment point, irrespective of its position in the box. If indifference curves are "kinked enough", the incompleteness of markets for contingent deliveries (the absence of trade) is then a direct consequence of preference for status quo which is exogenously imposed as a part of the definition of ambiguity aversion.

\section{The model and the main result}

The setting for our formal analysis is a model of a stylized two period finance economy which we call an $n$-financial asset economy with idiosyncracy. Households $(h=1, \ldots, H)$ trade assets in period 0 , before uncertainty is resolved, and consume the one (and only) good in period 1. The assets available for trade are claims on deliveries of the consumption good in period 1 .

There are two sources of uncertainty. First, there is some 'economic uncertainty': agents do not know their endowments tomorrow. An economic state of the world, $s, s=1,2$, is completely 
identified by the endowment vector for that state $\left(e_{1}^{s}, \ldots e_{h}^{s}, \ldots, e_{H}^{s}\right)$; where each component of the vector, $e_{h}^{s} \in \mathbb{R}_{+}$, gives a particular household's endowment of the consumption good in state $s$ (arising in period 1). We have restricted our analysis to the case of risk sharing across only two economic states, to make the argument as transparent as possible. Secondly, there is idiosyncratic financial uncertainty. An idiosyncratic state of the world completely characterizes the realization of the idiosyncratic components of payoffs of the available financial assets (described below); it is identified by the vector $t=\left(t_{1}, t_{2}, \ldots, t_{n}\right)$, where $t_{i} \in\{0,1\}, i \in\{1,2, \ldots, n\}$, and $n$ is the total number of financial assets. $\tau_{n}$ denotes the set of all $t$ 's, i.e., $\tau_{n} \equiv\{0,1\}^{n}$. Hence, to obtain a complete description of a state of the world, exhausting all uncertainty relevant to the model, the economic states $s$ must each be further partitioned into cells denoted $(s, t)$. A typical state of the world is denoted by the letter $\omega, \omega \in \Omega \equiv\left\{(1, t)_{t \in \tau_{n}},(2, t)_{t \in \tau_{n}}\right\}$.

The assets available for trade at date 0 are as follows:

1. Financial assets, $z^{i}, i=1, \ldots, n$, with payoffs that have idiosyncratic components. An asset $z^{i}$ yields a payoff of $y^{s}+y\left(t_{i}\right)>0$ units of the good; $s=1,2, t_{i} \in \tau \equiv\{0,1\} . y\left(t_{i}\right)$ is the idiosyncratic component, in the sense that it is independent of the realized economic state and independent of the realization of the payoff from any other financial asset $z^{j}$, where $j \neq i$. It is assumed that $y(1)>y(0)$ and that $y^{1} \neq y^{2}$. Price of an asset $z^{i}$ is denoted by $q_{n}^{z^{i}}$.

2. A safe asset, $b$, which delivers one unit of the good irrespective of the realized state of the world. Price of this security is normalized to 1.

A point behind modeling the asset structure as above is to ensure that in order to transfer resources across the two economic states the agents would have to rely on financial assets whose payoffs are affected by idiosyncratic shocks.

Prior to the resolution of uncertainty, agents are endowed with a common belief about the likelihood of state $\omega$. The (marginal) beliefs about particular idiosyncratic component $t_{i}$ are described by a capacity $\nu_{i}, \nu_{i}(0)+\nu_{i}(1) \leq 1$. To model the assumption that the realization of $t_{i}$ and $t_{j}$ are believed to be independent, the beliefs on $\tau_{n}$ are described by the independent product (defined in Appendix A) $\nu \equiv \bigotimes_{i=1}^{n} \nu_{i}$. For simplicity, we shall assume that $\nu_{i}\left(t_{i}=r\right)=$ $\nu_{j}\left(t_{j}=r\right)=\nu^{r}, r=0,1, i, j \in\{1, \ldots, n\}$. The belief on an economic state $s$ is given by $\pi(s)$. To make it transparent that it is the ambiguity of beliefs about the idiosyncratic realizations which is responsible for the possibility of no-trade in financial assets, and also to make the computation less tedious, we assume $\pi(1)+\pi(2)=1$. Finally, the common belief on $\Omega$ is given by the independent product $\pi \otimes \nu$.

Let $e_{h, n}^{\omega}$ and $x_{h, n}^{\omega}$ be $h$ 's endowment and consumption, respectively, in state $\omega=(s, t)$, given that the total number of financial assets in the economy is $n$. Note, the definition of an economic state implies $e_{h, n}^{(s, t)}=e_{h, n}^{\left(s, t^{\prime}\right)}$. Hence, we may use the notation $e_{h, n}^{s}$ as a complete description of state contingent endowment. Holding of the asset $b$ by $h$ is denoted $b_{h, n}$ and holding of the asset $z^{i}$ by $h$ is denoted $z_{h, n}^{i}$. Agent $h$ has a von-Neumann Morgenstern utility index $u_{h}: \mathbb{R}_{+} \rightarrow \mathbb{R}$, which is assumed to be strictly increasing, smooth and strictly concave. Furthermore, $u_{h}^{\prime}(0)=\infty$ and $e_{h, n}^{\omega}>0$ for all $h$ and all $\omega$.

$\mathcal{P}_{h n}$ which denotes the maximization program of agent $h$, is as follows:

$$
\begin{gathered}
\max _{b_{h, n}, z_{h, n}^{1}, \ldots, z_{h, n}^{n}} \mathbb{C E}_{\pi \otimes \nu} u_{h}\left(x_{h, n}^{s, t}\right) \\
\text { s.t. }\left\{\begin{array}{l}
b_{h, n}+\sum_{i=1}^{n} q_{n}^{z^{i}} z_{h, n}^{i}=0 \\
x_{h, n}^{s, t}-e_{h, n}^{s}=b_{h, n}+\sum_{i=1}^{n}\left(y^{s}+y\left(t_{i}\right)\right) z_{h, n}^{i}, s=1,2, t \in \tau_{n}
\end{array}\right.
\end{gathered}
$$


An equilibrium consists of a set of asset prices, $\mathbf{q}_{n} \equiv\left\{1, q_{n}^{z^{1}}, \ldots, q_{n}^{z^{n}}\right\}$, a set of asset holdings, $\left(\mathbf{b}_{n}, \mathbf{z}_{n}\right) \equiv\left\{\left(b_{h, n}, z_{h, n}^{1}, \ldots, z_{h, n}^{n}\right)_{h=1}^{H}\right\}$, and a consumption vector, $\mathbf{x}_{n} \equiv\left(x_{h, n}^{\omega}\right)_{h=1, \ldots, H ; \omega \in \Omega}$, such that, given $\mathbf{q}_{n}$ all agents solve $\mathcal{P}_{h n}$, and the asset markets clear, i.e.,

$$
\sum_{h} b_{h, n}=\sum_{h} z_{h, n}^{i}=0, \forall i \in\{1, \ldots, n\}
$$

and the consumption vector is feasible at each state, i.e., $\sum_{h} x_{h, n}^{\omega}=\sum_{h} e_{h, n}^{\omega}$. Notice, a tuple $\left(\mathbf{q}_{n},\left(\mathbf{b}_{n}, \mathbf{z}_{n}\right)\right)$ uniquely pins down the equilibrium, hence we may denote an equilibrium of an $n$-financial asset economy using such a tuple.

In interpreting various aspects of the model it helps to bear in mind the economic issue the model has been formulated to examine, which is, how economic agents may share risks, inherent to their labor/human capital endowment, by trading in financial markets. Hence, as it appears in the model, a household's endowment income is distinct from the household's income obtained from the ownership of assets. Portfolio income is the instrument the household is allowed to use to absorb the shocks it faces in its endowed income. But the instrument is not a perfect one. The presence of idiosyncratic risk embodies the notion that payoff from a financial asset is not only affected by some of the same shocks that affect individual households' endowment income and common to many assets but also by risks specific to each asset. While most firms' profits are naturally affected by aggregate or sectorial demand shifts and supply shocks, other factors, more idiosyncratic to the firm, do typically matter ${ }^{6}$. Finally, notice, we have assumed that the assets are in zero net supply. This implies that the asset trading our analysis applies to include all manner of trade in corporate bonds ${ }^{7}$; but for general assets (e.g., equities) the analysis is (formally) restricted to those trades which involve one side of the market going short. The main point of the assumption is that it allows us the abstraction to study how an agent may use a financial asset (say, an equity) to share the risk in his exogenously endowed income: by going short on an asset he issues contingent claims on his risky income, thereby, trading out his risk. To fix ideas, it might help to refer back to the example of X, the Detroit drug-store owner. X would be very representative of the agents in our model presented above. Think of the economic states 1 and 2 as states defined by shocks to X's income from his drug-store. X may hedge his income shock by trading in a "safe" asset, such as a treasury bond, and financial assets, such as corporate bonds/equities issued by the various automobile and ancillary firms located in and around Detroit. Payoffs to each such financial asset is affected by the same income shock that affects X's drug-store profits. In addition, each financial asset is also affected by shocks idiosyncratic to the issuing firm. Assuming, the firms' profits and drug-store profits are affected in the same direction by the income shock, X's hedging strategy would be, presumably, to take a short position on a portfolio of the available financial assets while simultaneously going long on the treasury bond. Our analysis, in effect, compares how such a strategy would fare in an SEU world and in a CEU world.

\footnotetext{
${ }^{6}$ For instance, suppose a firm introduces a new product line, an innovation, into the market. In such a case, typically, it is not just the shocks commonly affecting firms in the same trade that will affect the sales of the new product but also more (brand) specific elements, e.g. whether (or not) the innovation has a 'special' appeal for the consumers. Another example of idiosyncratic shocks, are shocks to firms' internal organizational capabilities.

${ }^{7}$ In this context it is worth noting that it is reported almost $70 \%$ of corporate borrowing in the U.S. is through bonds. Default rates on bonds are also significant. Financial Times, 13 October 1998, in its report headlined "US corporate bond market hit," notes, "the rate of default on US high-yield bonds was running at $10 \%$ in the early 1990s...today the default rate is hovering around $3 \%$ but creeping higher".
} 
Formally, the analysis compares equilibrium allocations across two cases: one, where beliefs about idiosyncratic outcome is unambiguous $\left(\nu^{0}+\nu^{1}=1\right)$, and the other where beliefs about the idiosyncracy is ambiguous $\left(\nu^{0}+\nu^{1}<1\right)$. In order to make the comparison stark, the analysis will relate the two cases to two benchmarks. One benchmark is a complete market economy which we call an economy without idiosyncracy, that is, an economy which is identical to the $n$-financial asset economy with idiosyncracy described in the last section in every respect except that there is only a single financial asset $z$ which pays off $y^{s}+\mathbb{E}_{\nu} y(t) \equiv \bar{y}^{s}$ units in the economic states $s=1,2$. Correspondingly, $q^{z}$ denotes the price of $z$ and $z_{h}$ denotes the amount held by household $h$. (Note, when denoting endogenous variables in the economy without idiosyncracy we may omit the subscript $n$.) The second benchmark is an incomplete market economy which is identical to the $n$-financial asset economy with idiosyncracy in every respect except that the only asset available is the safe asset. The following Lemma simplifies the analysis greatly.

Lemma Let $\left(\mathbf{q}_{n},\left(\mathbf{b}_{n}, \mathbf{z}_{n}\right)\right)$ be an equilibrium of the $n$-financial assets economy with idiosyncracy. Suppose $\nu^{0}+\nu^{1} \leq 1$. Then, $z_{h, n}^{i}=z_{h, n}^{i^{\prime}}, \forall i, i^{\prime} \in\{1, \ldots, n\}, \forall h \in\{1, \ldots, H\}$.

According to the Lemma, at an equilibrium, agents will hold all the financial assets in the same proportion. This is essentially a consequence of the fact that agents are risk averse and that the $n$ financial assets are simply 'independent replicas'. Let $\tilde{z}_{n}$ denote an unit of a portfolio composed of $\frac{1}{n}$ unit of the asset $z^{i}, i=1, \ldots, n ; \tilde{z}_{h, n}$ is the amount held of this portfolio by $h$ and $\tilde{q}_{n}$ is the price of an unit of this portfolio. Given the Lemma, we may assume, without loss of generality, that it is only the asset $\tilde{z}_{n}$, instead of the individual assets $z^{i}$, that is available for trade in the economy. Hence, an equilibrium of an $n$-financial assets economy with idiosyncracy, $\left(\mathbf{q}_{n},\left(\mathbf{b}_{n}, \mathbf{z}_{n}\right)\right)$, may equivalently be denoted by the tuple $\left(\widetilde{\mathbf{q}}_{n},\left(\mathbf{b}_{n}, \widetilde{\mathbf{z}}_{n}\right)\right), \widetilde{\mathbf{q}}_{n} \equiv\left\{1, \tilde{q}_{n}\right\}$ and $\left(\mathbf{b}_{n}, \widetilde{\mathbf{z}}_{n}\right) \equiv\left\{\left(b_{h, n}, \tilde{z}_{h, n}\right)_{h=1}^{H}\right\}$.

The above characterization of the equilibrium in turn facilitates a simple definition of what it means to satisfy the conditions of equilibrium when $n$ is arbitrarily large. We say $\left(\widetilde{\mathbf{q}}_{\infty},\left(\mathbf{b}_{\infty}, \widetilde{\mathbf{z}}_{\infty}\right), \mathbf{x}_{\infty}\right)$ satisfies the conditions of equilibrium of the $n$-financial assets economy with idiosyncracy where $n$ is arbitrarily large, i.e., $n \rightarrow \infty$ if $^{8}$ :

1. Given $\widetilde{\mathbf{q}}_{\infty},\left(\left(\mathbf{b}_{\infty}, \widetilde{\mathbf{z}}_{\infty}\right), \mathbf{x}_{\infty}\right)$ is a solution to the problem $\widetilde{\mathcal{P}}_{h, \infty}$ defined as follows:

$$
\begin{gathered}
\max \mathbb{C E}_{\pi \otimes \nu} u_{h}\left(x_{h, \infty}^{s, t}\right) \\
\text { s.t. }\left\{\begin{array}{l}
b_{h, \infty}+\tilde{q}_{\infty} \tilde{z}_{h, \infty}=0 \\
x_{h, \infty}^{s, t}-e_{h, \infty}^{s}=b_{h, \infty}+\tilde{z}_{h, \infty}\left[\lim _{n \rightarrow \infty} \frac{\sum_{i=1}^{n}\left(y^{s}+y\left(t_{i}\right)\right)}{n}\right], \\
s=1,2, \text { with probability } 1,
\end{array}\right.
\end{gathered}
$$

2. $\sum_{h} b_{h, \infty}=\sum_{h} \widetilde{z}_{h, \infty}=0$, and the consumption vector is feasible at each state, i.e., $\sum_{h} x_{h, \infty}^{\omega}=\sum_{h} e_{h, \infty}^{\omega}$ with probability 1 .

Theorem Suppose $\nu^{0}+\nu^{1}=1$. Then $\left(\widetilde{\mathbf{q}}_{\infty},\left(\mathbf{b}_{\infty}, \widetilde{\mathbf{z}}_{\infty}\right)\right)$ satisfies the conditions of equilibrium of the $n$-financial assets economy with idiosyncracy where $n$ is arbitrarily large, if and only if, $\left(\widetilde{\mathbf{q}}_{\infty},\left(\mathbf{b}_{\infty}, \widetilde{\mathbf{z}}_{\infty}\right)\right)$ describes an equilibrium of an economy without idiosyncracy, wherein the price of a unit of $z$ is equal to $\tilde{q}_{\infty}$, and a household's holding of the asset $z, z_{h}$, is equal to $\tilde{z}_{h, \infty}$.

\footnotetext{
${ }^{8}$ Werner (1997) considers a finance economy of which this is just a special case. There are standard arguments that ensure the existence of equilibria of such economies (op.cit., pp.100).
} 
The theorem shows that equilibrium allocations of the $n$-financial assets economy with idiosyncracy are essentially identical to that of the economy without idiosyncracy, in which financial markets are complete, provided the number of available financial assets is large enough and agents' beliefs are unambiguous. The result follows from an application of the usual diversification principle stating that in the limit idiosyncracies are 'washed away', in conjunction with, the assumption that $y^{1} \neq y^{2}$. However, if the model of the $n$-financial assets economy with idiosyncracy were to be reconsidered with the sole amendment that beliefs about idiosyncracies are ambiguous, i.e., $\nu^{0}+\nu^{1}<1$, then the result no longer holds. In such an economy, however large the $n$, given sufficient ambiguity, the equilibrium allocation is bounded away from Pareto optimal risk-sharing. The allocation actually coincides with the allocation of an incomplete market economy in which it is impossible to transfer resources between states 1 and 2, as we show in our main theorem, below. But, first, we present an example to convey an intuition for the result.

Example 1 Consider a 2-period finance economy with two risk averse agents, $h=1,2$, and two economic states. There are two assets available, $b$ and $z . b$ is a safe asset; it delivers one unit of the good in each of the two economic states. The payoff of $z$ in state $(s, t)$ is $y^{s}+y(t)$, $s=\alpha, \beta ; t=0,1$. Fix, $y^{\alpha}=1, y^{\beta}=2, y(0)=0, y(1)=2$.

First consider the case where $\nu^{0}+\nu^{1}=1$. The model reduces to a standard incomplete market equilibrium with two assets and four states, in which, for 'generic' endowments, there is trade, i.e., some partial insurance among agents ${ }^{9}$. Next, suppose, to simplify matters drastically, that $\nu^{0}=\nu^{1}=0$. Consider an agent $h$ contemplating buying the uncertain asset at a price $q^{z}$, given the safe asset is priced $q^{b}=1$. $h$ may buy $z_{h}$ units of the uncertain asset and take a position $b_{h}$ in the safe asset such that $b_{h}+q^{z} z_{h}=0$. His utility functional is then given by:

$$
\begin{aligned}
& \mathbb{C E}_{\pi \otimes \nu} u_{h}\left(e_{h}^{s}+z_{h}\left(y^{s}+y(t)\right)+b_{h}\right) \\
= & u_{h}\left(e_{h}^{\alpha}+z_{h}\left(y^{\alpha}+y(0)\right)+b_{h}\right) \pi(\alpha)\left(1-\nu^{1}\right)+u_{h}\left(e_{h}^{\alpha}+z_{h}\left(y^{\alpha}+y(1)\right)+b_{h}\right) \pi(\alpha) \nu^{1} \\
& +u_{h}\left(e_{h}^{\beta}+z_{h}\left(y^{\beta}+y(0)\right)+b_{h}\right) \pi(\beta)\left(1-\nu^{1}\right)+u_{h}\left(e_{h}^{\beta}+z_{h}\left(y^{\beta}+y(1)\right)+b_{h}\right) \pi(\beta) \nu^{1}
\end{aligned}
$$

Once we substitute in $\nu^{1}=0$, it is clear from the above functional that the payoff matrix the agent (as a buyer of $z$ ) will consider is:

$$
\left[\begin{array}{ll}
1 & 1 \\
1 & 2
\end{array}\right]
$$

If $q^{z} \geq 2$, any balanced portfolio with $z_{h}>0$ yields negative payoffs and is therefore not worth buying. Thus, an agent will wish to buy the uncertain asset only if $q^{z}<2$. Next consider an agent $h^{\prime}$ who contemplates going short on asset $z$. His utility functional is therefore:

$$
\begin{aligned}
& \mathbb{C E}_{\pi \otimes \nu} u_{h^{\prime}}\left(e_{h^{\prime}}^{s}+z_{h^{\prime}}\left(y^{s}+y(t)\right)+b_{h^{\prime}}\right) \\
= & u_{h^{\prime}}\left(e_{h^{\prime}}^{\alpha}+z_{h^{\prime}}\left(y^{\alpha}+y(0)\right)+b_{h^{\prime}}\right) \pi(\alpha) \nu^{0}+u_{h^{\prime}}\left(e_{h^{\prime}}^{\alpha}+z_{h^{\prime}}\left(y^{\alpha}+y(1)\right)+b_{h^{\prime}}\right) \pi(\alpha)\left(1-\nu^{0}\right) \\
& +u_{h^{\prime}}\left(e_{h^{\prime}}^{\beta}+z_{h^{\prime}}\left(y^{\beta}+y(0)\right)+b_{h^{\prime}}\right) \pi(\beta) \nu^{0}+u_{h^{\prime}}\left(e_{h^{\prime}}^{\beta}+z_{h^{\prime}}\left(y^{\beta}+y(1)\right)+b_{h^{\prime}}\right) \pi(\beta)\left(1-\nu^{0}\right)
\end{aligned}
$$

Notice now the functional is dependent on $\nu^{0}$ since the agent is going short, i.e., $z_{h^{\prime}}<0$. Substituting $\nu^{0}=0$, we find the payoff matrix the agent $h^{\prime}$ will consider:

$$
\left[\begin{array}{ll}
1 & 3 \\
1 & 4
\end{array}\right]
$$

${ }^{9}$ This has to be qualified since there exists some non-generic constraints among endowments in different states, namely $e_{h}^{s, t}=e_{h}^{s, t^{\prime}} \equiv e_{h}^{s}$. 
For $q^{z} \leq 3$ any balanced portfolio with $z_{h^{\prime}}<0$ yields negative payoffs. Thus, an agent will wish to sell the risky asset only if $q^{z}>3$. Thus, buyers of asset 1 will not want to pay more than 2 , while sellers will not sell it for less than 3 . Hence, there does not exist an equilibrium price such that agents will have a non-zero holding of the uncertain asset.

Next, consider another extreme, a case in which ambiguity appears only on the economic states while the agents are able to assess (additive) probabilities for the idiosyncratic states. In fact, to keep matters stark, assume $\pi(\alpha)=\pi(\beta)=0$, though the additive probability on idiosyncratic states is arbitrary, simply ensuring that $\nu^{0}+\nu^{1}=1$. Suppose that, for agent $h$, $e_{h}^{\alpha}>e_{h}^{\beta}$. Then, for $z_{h} \in(-\varepsilon, \varepsilon)$, for $\varepsilon$ small enough,

$$
\begin{aligned}
& \mathbb{C E}_{\pi \otimes \nu} u_{h}\left(e_{h}^{s}+z_{h}\left(y^{s}+y(t)\right)+b_{h}\right) \\
= & \nu^{0} u_{h}\left(e_{h}^{\beta}+z_{h}\left(y^{\beta}+y(0)\right)+b_{h}\right)+\nu^{1}\left(e_{h}^{\beta}+z_{h}\left(y^{\beta}+y(1)\right)+b_{h}\right)
\end{aligned}
$$

since for $z_{h}$ small enough $e_{h}^{\alpha}+z_{h}\left(y^{\alpha}+y(t)\right)+b_{h}>e_{h}^{\beta}+z_{h}\left(y^{\beta}+y(t)\right)+b_{h}$. Hence, $z_{h}=0$ if and only if

$$
q^{z}=y^{\beta}+\nu^{0} y(0)+\nu^{1} y(1)
$$

(the fact that endowments and the utility function do not appear in this expression is due to the extreme form of ambiguity assumed, i.e., a maximin behavior). Thus, the only candidate for a no-trade equilibrium price is $q^{z}=y^{\beta}+\nu^{0} y(0)+\nu^{1} y(1)$. Now, assume that for at least one other agent, the order of the endowment is reversed, i.e., $e_{h}^{\beta}>e_{h}^{\alpha}$, then a computation similar to the one above shows that such agents will not want to trade the risky asset if and only if

$$
q^{z}=y^{\alpha}+\nu^{0} y(0)+\nu^{1} y(1)
$$

Hence, if both types of agents are present in the economy, trade will occur as $y^{\alpha} \neq y^{\beta}$. If we were not to assume the extreme maximin form of preferences but that $\pi(\alpha)+\pi(\beta)<1$ with, say, $\pi(\alpha)>0$ and $\pi(\beta)>0$, the no trade price for agent $h$ (say with $e_{h}^{\alpha}>e_{h}^{\beta}$ ) depends on his initial endowment and utility function (i.e., relative attitude to risk). In that case, even if endowments of all agents were comonotonic (i.e., $e_{h}^{\alpha} \geqslant e_{h}^{\beta}$ for all $h$ ) there would $\underline{\text { not }}$ exist, for the generic endowment vector, an asset price $q^{z}$ that would support no-trade as an equilibrium of this economy.

The two more significant ways in which the main theorem, below, generalizes the demonstration in Example 1 are: one, it shows that no-trade obtains even when beliefs have a degree of ambiguity strictly less than 1; two, it allows for any arbitrary number of financial assets, in particular, for $n \rightarrow \infty$. We consider the intuition for each of these generalizations in turn. First, consider a 2-(economic)-state, 2 agent, 1-financial asset (and 1 safe asset) economy with idiosyncracy, in which the financial asset's payoffs are as in Example 1. Consider an agent thinking of buying the financial asset. The maximum payoff he expects in any economic state is $2+0 \times\left(1-\nu^{1}\right)+2 \times \nu^{1} \equiv V(B)$, the amount he expects in state $\beta$. This implies, whatever his utility function, whatever his endowment vector, whatever his beliefs about the economic uncertainty, he will not want to buy the asset for more than $V(B)$. Now, instead, if an agent were to go short with the asset, the least he expects to have to repay in any economic state is $1+0 \times \nu^{0}+2 \times\left(1-\nu^{0}\right) \equiv V(S)$, and therefore, will not want to sell the asset if the price is less than this. Clearly, if $\nu^{0}$ and $\nu^{1}$ were small enough, $V(B)<V(S)$. Therefore, if $\nu^{0}$ and $\nu^{1}$ were small enough, agents will not trade in the financial asset.

Intuition about the second bit of generalization is difficult to obtain without some understanding of how the law of large numbers works for non-additive beliefs. Specifically, 
let us consider an i.i.d. sequence $\left\{X_{n}\right\}_{n \geq 1}$ of $\{0,1\}$-valued random variables. Suppose, $\nu\left(\left\{X_{n}=0\right\}\right)=\nu\left(\left\{X_{n}=1\right\}\right)=\frac{1}{4}$ for all $n \geq 1$. As is usual with laws of large numbers, the question is about the limiting distribution of the sample average, $\frac{1}{n} \sum_{i=1}^{n} X_{i}$. The law ${ }^{10}$ implies:

$$
\nu\left(\frac{1}{4} \leq \liminf _{n \rightarrow \infty} \frac{1}{n} \sum_{i=1}^{n} X_{i} \leq \limsup _{n \rightarrow \infty} \frac{1}{n} \sum_{i=1}^{n} X_{i} \leq \frac{3}{4}\right)=1 .
$$

This shows that the DM has a probability 1 belief that the limiting value of the sample average lies in the (closed) interval $\left[\frac{1}{4}, \frac{3}{4}\right]$. However, unlike in the case of additive probabilities, the DM is not able to further pin down its value. Thus, even with non-additive probabilities the law of large numbers works in the usual way, in the sense that here too the tails of the distribution are 'canceled out' and the distribution 'converges on the mean'. But of course here, given that the DM's knowledge is consistent with more than one prior, there is more than one mean to converge on; hence, the convergence is to the set of means corresponding to the set of priors consistent with the DM's knowledge. Hence, a CEU maximizer whose (ex post) utility is increasing in $X$ (e.g., when the DM is a buyer of an asset with payoff $X$ ) will behave as if the convergence of the sample average occurred at $\frac{1}{4}$, the lower boundary of the interval, while a DM whose utility is increasing in $-X$ (e.g., when the DM is a seller of an asset with payoff $X$ ) will behave as if the convergence of the sample average occurred at, $\frac{3}{4}$, the upper boundary of the interval.

Now we can complete our intuition for the main result. Consider a modification of the simplified financial economy of Example 1 such that, ceteris paribus, there are now $n$ fold replicas of the financial asset, $n \rightarrow \infty$. We consider trade between 'two' assets, one the safe asset and the other the 'portfolio' asset, containing each of the independent replica assets in equal proportion. The law of large numbers result, explained above, implies that any agent contemplating going long on the portfolio asset will behave as if a unit of the portfolio will payoff $y^{s}+\left[0 \times\left(1-\nu^{1}\right)+2 \times \nu^{1}\right]$ with probability 1 in economic state $s$ while an agent contemplating going short will behave as if a unit of the portfolio will payoff $y^{s}+\left[0 \times \nu^{0}+2 \times\left(1-\nu^{0}\right)\right]$ with probability 1 in economic state $s$. Hence, exactly the same argument as before applies: for $\nu^{0}$ and $\nu^{1}$ sufficiently small, $V(B)<V(S)$ and there will not be any trade in the portfolio. The important insight here is that while agents are fully aware that a 'well diversified' portfolio 'averages out' the idiosyncracies, they only have an imprecise knowledge of what it averages out to.

Another important point demonstrated in Example 1, as modified above, is how equilibrium risk sharing is affected by ambiguity aversion. If $1-\nu^{0}-\nu^{1}>\frac{1}{2}$, then the equilibrium allocation is necessarily not Pareto optimal unless endowments are, no matter how large the value of $n$. Consider an economy, $\mathcal{E}$, which is the same as in the original example except that there is only one financial asset available in this economy, the safe asset $b$. Given ambiguity is greater than $\frac{1}{2}$, there is no trade in the portfolio of uncertain assets in the economy in (the modified) Example 1, hence an equilibrium allocation of this economy is an equilibrium allocation of $\mathcal{E}$. $\mathcal{E}$ has two states, $\alpha$ and $\beta$, but one asset, and therefore, is an incomplete markets economy with sub-optimal risk sharing.

We now state our main result:

Main Theorem Consider the $n$-financial assets economy with idiosyncracy. Let $y^{\underline{s}} \equiv \min _{s}\left\{y^{s}\right\}$

\footnotetext{
${ }^{10}$ Laws of large numbers for ambiguous beliefs have been studied by, among others, Walley and Fine (1982), Marinacci (1996) and Marinacci (1999). Appendix A contains a formal statement of the version we apply. This version was, essentially, originally proved in Walley and Fine (1982). The statement given here is from Marinacci (1996), Theorem 7.7. However, the result is a direct implication of the more general Theorem 15 in Marinacci (1999).
} 
and $y^{\bar{s}} \equiv \max _{s}\left\{y^{s}\right\}$ and suppose that $y^{\bar{s}}-y^{\underline{s}}>y(1)-y(0)$. Then there exists an $\overline{\mathcal{A}}, 0<\overline{\mathcal{A}}<1$, such that if $1-\nu^{0}-\nu^{1}>\overline{\mathcal{A}}, \tilde{z}_{h, n}=0$ for all $h \in\{1, \ldots, H\}$ and $x_{n}^{s, t}=x_{n}^{s, t^{\prime}}$, $s=1,2, t \neq t^{\prime}$ at every equilibrium $\left(\mathbf{q}_{n},\left(\mathbf{b}_{n}, \mathbf{z}_{n}\right)\right)$, for all $n \in \mathbb{N}$, including, $n \rightarrow \infty$.

Stated differently, this says that if the range of variation of the idiosyncratic component of the financial asset is greater than the range of variation due to the economic shocks, if the beliefs over the idiosyncratic states are ambiguous enough, and if agents are ambiguity averse, then irrespective of the utility functions of the agents and the endowment vector, the equilibrium of an $n$-financial assets economy with idiosyncracy is an equilibrium of the economy with one safe asset, i.e., an economy with incomplete markets, since the financial assets are not traded in equilibrium, whatever the value of $n$. Notice further, if the conditions described in the theorem are met, then for a generic endowment, an equilibrium allocation of the $n$ financial assets economy with idiosyncracy is necessarily not Pareto optimal. This follows simply from the understanding that an equilibrium allocation of the $n$-financial assets economy with idiosyncracy, given the conditions of the theorem, is an equilibrium of the economy with one safe asset. The latter economy is an incomplete market economy in which it would not be possible to transfer resources between states 1 and 2 .

The significant sufficient condition to ensure no-trade, irrespective of the utility functions of the agents and the endowment vector, is that $y^{\bar{s}}-y^{\underline{s}}>y(1)-y(0)$. The bound follows from the expression for $\overline{\mathcal{A}}, \overline{\mathcal{A}}=\frac{y^{s}-y^{\underline{s}}}{y(1)-y(0)}$, constructed in the proof of the main theorem. Notice, $\overline{\mathcal{A}}$ is the supremum among the values of ambiguity required for no trade, across all the possible combinations of parameters of utilities or endowments, and is independent of any parameter of utility or endowment. So, typically, the ambiguity required for no trade be less than $\overline{\mathcal{A}}$; further, no trade will result even if $y(1)-y(0)<y^{\bar{s}}-y^{\underline{s}}$. Also, the required ambiguity will be greater, greater the risk aversion and/or riskiness of the endowment (see example 3 in Mukerji and Tallon (1999)).

One might be tempted to conjecture that results of the paper may be replicated by simply assuming heterogeneous beliefs among agents. Or to conjecture, since with incomplete markets comonotonicity of equilibrium allocations is in general broken so that different (CEU) agents would evaluate their prospects using different (effective) probabilities, that adding CEU agents might "worsen" incompleteness even in the absence of idiosyncratic risks. Both conjectures are, however, false. What is at work in obtaining no-trade is not that different agents have different beliefs but that any given agent behaves as if he evaluates the two different actions, going short and going long, with different (probabilistic) beliefs. Neither does market incompleteness, in the absence of idiosyncratic risk, make for this peculiarity and therefore does not, in and of itself, lead to no trade. We illustrate this with the following example.

Example 2 Suppose there are $S$ states, $H$ agents, one safe asset and one risky asset that pays off $y^{s}$ unit of the good in state $s$. Agent $h$ 's budget constraints are (we normalize the price of the safe asset in the first period as well as the price of the good in all states to be equal to 1):

$$
\left\{\begin{aligned}
b_{h}+q z_{h} & =0 \\
x_{h}^{s} & =e_{h}^{s}+\left(y^{s}-q\right) z_{h} \quad s=1, \ldots, S
\end{aligned}\right.
$$

Claim Assume that there are no pairs of states $s$ and $s^{\prime}$ such that $y^{s} \neq y^{s^{\prime}}$ and $e^{s}=e^{s^{\prime}}$. Then, there exists a unique price $q_{h}$ such that $z_{h}^{*}\left(q_{h}\right)=0$.

Proof. Assume w.l.o.g. that $e_{h}^{1} \leq e_{h}^{2} \leq \cdots \leq e_{h}^{S}$. Since by assumption $e_{h}^{s}=e_{h}^{s^{\prime}} \Rightarrow y^{s}=y^{s^{\prime}}$, there exists $\varepsilon>0$ such that for all $z_{h} \in(-\varepsilon, \varepsilon)$ :

$$
e_{h}^{1}+\left(y^{1}-q\right) z_{h} \leq e_{h}^{2}+\left(y^{2}-q\right) z_{h} \leq \cdots \leq e_{h}^{S}+\left(y^{S}-q\right) z_{h}
$$


Let $\Delta\left(z_{h}\right)$ be the set of probability measures in $\mathcal{C}(\nu)$ that minimize $\mathbb{E}_{\mu \in \mathcal{C}(\nu)} u_{h}\left(e_{h}+\left(y^{s}-q\right) z_{h}\right)$, i.e., $\Delta\left(z_{h}\right)=\left\{\left(\mu^{1}, \ldots, \mu^{S}\right) \in \mathcal{C}(\nu) \mid \mathbb{E}_{\mu} u_{h}\left(e_{h}+\left(y^{s}-q\right) z_{h}\right)=\mathbb{E}_{\nu} u_{h}\left(e_{h}+\left(y^{s}-q\right) z_{h}\right)\right\}$. Observe that if $\mu, \mu^{\prime} \in \Delta\left(z_{h}\right)$ are different, then they must disagree on those states where consumption is identical, or, said differently (given the order we adopted on $h$ 's endowment):

$$
\begin{aligned}
e_{h}^{s}+\left(y^{s}-q\right) z_{h} & \neq e_{h}^{s^{\prime}}+\left(y^{s^{\prime}}-q\right) z_{h} \forall s^{\prime} \neq s \\
& \Rightarrow \mu^{s}=\mu^{\prime s}=\nu(\{s, \ldots, S\})-\nu(\{s+1, \ldots, S\})
\end{aligned}
$$

Hence, $z_{h}=0$ is optimal at price $q_{h}$ if and only if there exists $\mu \in \Delta(0)$ such that:

$$
q=q_{h} \equiv \frac{\sum_{s} \mu^{s} y^{s} u_{h}^{\prime}\left(e_{h}^{s}\right)}{\sum_{s} \mu^{s} u_{h}^{\prime}\left(e_{h}^{s}\right)}
$$

Recall now that probability measures in $\Delta(0)$ can differ only on those states in which the endowment is constant. Since, by assumption, $e_{h}^{s}=e_{h}^{s^{\prime}} \Rightarrow y^{s}=y^{s^{\prime}}$, one obtains, $\mathbb{E}_{\mu}\left[y^{s} u_{h}^{\prime}\left(e_{h}^{s}\right)\right]=$ $\mathbb{E}_{\mu^{\prime}}\left[y^{s} u_{h}^{\prime}\left(e_{h}^{s}\right)\right]$ for all $\mu, \mu^{\prime} \in \Delta(0)$. Since $\mathbb{E}_{\mu} u_{h}^{\prime}\left(e_{h}^{s}\right)=\mathbb{E}_{\mu^{\prime}} u_{h}^{\prime}\left(e_{h}^{s}\right)$ for all $\mu, \mu^{\prime} \in \Delta(0), q_{h}$ as defined above is unique.

We just established that there is only one price $q_{h}$, defined in the proof above, such that at this price, agent $h$ optimally wants a zero position in the risky asset. Now, unless the endowment allocation is Pareto optimal, $q_{h} \neq q_{h^{\prime}}$. Hence, at an equilibrium, trade on the market for the risky asset will be observed. This establishes that, "generically", in order for $z_{h}=0$ for all $h$ to be an equilibrium of the model, there must be pairs of states $s, s^{\prime}$ such that $e_{h}^{s}=e_{h}^{s^{\prime}}$ for all $h$ and $y^{s} \neq y^{s^{\prime}}$; in other words, an idiosyncratic element is necessary to obtain no trade.

Before we close this section, we attempt to clarify further how our main result adds to the findings in the related literature. In Example 2, inspite of an incomplete markets environment, inspite of CEU agents, no trade fails to materialize because each agent has a unique price at which he takes a zero position in the asset, and in general, this price is different for different agents. Dow and Werlang (1992) may be read as an exercise in purely deriving the demand function for a risky asset, given an initial riskless position. By putting together two Dow and Werlang agents one does obtain an economy where an equilibrium may be defined, but given that such agents' endowments are riskless, agents do not have any risks to share in such an economy. Hence, simply "completing" the Dow and Werlang exercise to obtain an equilibrium model does not allow one to investigate the question addressed in the present paper, which is, whether ambiguity aversion affects risk sharing possibilities in the economy. And, as explained in the previous section, even if we were to make the simple further extension of allowing uncertain endowments, given complete markets, we will find ambiguity has no effect. Finally, as Example 2 demonstrates, an even further extension of allowing market incompleteness does not provide the answer either. Evidently, one has to move further afield from the Dow and Werlang analysis to address our question.

Epstein and Wang (1994) significantly generalized the Dow and Werlang (1992) result to find that price intervals supporting the zero position occurred (in equilibrium) if there were some states across which asset payoffs differ while endowments remain identical. The intuition for this is as follows. To obtain a range of supporting prices for the zero position, there must occur a "switch" in the effective probability distribution precisely at the zero position. That is, depending on whether he takes a position $+\epsilon$ or $-\epsilon$ away from $0, \epsilon$ howsoever small, the agent evaluates his position using a different probability. For this to happen, the agent's ranking of states (according to his consumption) must switch depending exclusively on whether he takes a positive or negative position on the asset. Hence, there must be at least two states for which even the smallest movement away from the zero position would cause a difference in the ranking 
of the states depending on which side of zero one moves to. Clearly, this may only be true if the endowment were constant across the two states while the asset payoff were not.

The clarification obtained in Epstein and Wang (1994) of the condition that enables multiple price supports to emerge, was the point of inspiration for the research reported in the present paper. Indeed, the condition of Epstein and Wang (1994) is one of the two conditions we apply to define idiosyncratic risk. Where the present paper has gone further and what, in essence, is its contribution, is in finding conditions for an economy wherein the agents' price intervals overlap in such a manner that every equilibrium of the economy involves no-trade in an asset, and more importantly, conditions under which ambiguity aversion demonstrably "worsens" risk sharing and incompleteness of markets. These are issues that were neither addressed nor even raised in Epstein and Wang (1994), formally or informally, and understandably so, since the principal model in that paper was the Lucas (1978) pure exchange economy amended to include ambiguity averse beliefs. This is a model with a single representative agent, or equivalently, a number of agents with identical preferences and endowments. In an equilibrium of such an economy, trade and risk sharing is trivial since agents will consume their endowments; endowments are, by construction, Pareto optimal ${ }^{11}$.

Kelsey and Milne (1995) extends the equilibrium arbitrage price theory (APT) by allowing for various kinds of non-expected utility preferences. One of the cases they consider is the CEU model. The model in the present paper may be thought of as a special case of the equilibrium APT framework: what are labeled as factor risks in APT are precisely what we call economic states and idiosyncratic risk is present in both models though in our model the idiosyncratic risk has a simpler structure in that there are only two possible idiosyncratic states corresponding to each asset. Only a special case of CEU preferences is investigated by Kelsey and Milne (1995): their Assumption 3.3 allows non-additive beliefs only with respect to factor risks; idiosyncratic risk is described only by additive probabilities (see Assumption 3.3, the Remark following the assumption and footnote 2). The formal result of their analysis appears in Corollary 3.1 and shows, given the qualifications, the usual APT result continues to hold: diversification may proceed as usual, idiosyncratic risk disappears in the limit as the number of assets tend to infinity and the price of any asset is, consequently, a linear function of factor risks. This formal result is readily understandable given our analysis. As is repeatedly stressed upon in the present paper, what drives our result is the non-additivity of beliefs over the idiosyncratic states. While it is not necessary that ambiguity aversion be restricted to idiosyncratic states for our result to hold, it is necessary that there be some ambiguity about idiosyncracies. The no-trade result fails if ambiguity is merely restricted to economic states, as we explained in the latter part of Example 1 and in Example 2. With ambiguity only on economic states, ambiguity aversion has no bite, irrespective of whether there is only a single asset or infinitely many and hence diversification proceeds as with SEU. Hence, their result would not obtain without the restriction imposed by (their) Assumption 3.3. Our analysis therefore warns against informally extrapolating the Kelsey and Milne (1995) result to think that diversification would proceed as usual even when the special circumstances of Assumption 3.3 does not hold (i.e., the ambiguity is not restricted to economic states but occurs more generally over the state space). Further, it would appear to be a compelling description of the economic environment to assume, if an agent is at all ambiguity averse, the agent will be ambiguity averse about an idiosyncratic risk. By definition, such a risk is unrelated to his own income risk and the macroeconomic environment; the risk stems from the internal workings of a particular firm, something about which the typical agent is likely to have little knowledge of.

\footnotetext{
${ }^{11}$ Section 3.4 of Epstein and Wang (1994), presents an example of an economy with heterogeneous agents. But, in this model markets are assumed to be complete, and hence, risk sharing continues to be efficient (Pareto optimal), as is explicitly observed by the authors.
} 
It is well-known that it is possible to define more than one notion of independence for nonadditive beliefs. Ghirardato (1997) presents a comprehensive analysis of the various notions. As Ghirardato notes (pp. 263), the problem of defining an independent product has been studied, previous to Ghirardato's investigation, by Hendon, Jacobsen, Sloth, and Tranaes (1996), Gilboa and Schmeidler (1989) and Walley and Fine (1982). The definition invoked in the present paper, suggested by Gilboa and Schmeidler (1989) and Walley and Fine (1982), is arguably the more prominent in the literature. However, the formal analysis in the present paper, given the primitives of our model, does not hinge on this particular choice of the notion of independence. An important finding of Ghirardato's analysis was that the proposed specific notions of independent product give rise to a unique product for cases in which marginals have some additional structural properties. The capacity we use in our model is a product of an additive probability and $n$ two-point capacities ( $\nu$ consists of two points, $\nu^{0}$ and $\nu^{1}$ ). A two-point capacity is, of course, a convex capacity and (trivially) a belief function. As is explicit in Theorems 2 and 3 in Ghirardato (1997), if marginals satisfy the structural properties the marginals we use do, then uniqueness of product capacity obtains. That is, irrespective of which of the two definitions of independence is adopted, the one suggested by Hendon et al. or the one we use, the computed product capacity is the same. The law of large numbers that we use formally invokes the Gilboa-Schmeidler notion (see Theorem 15 and Section 7.2 in Marinacci (1999)). Since both notions of independence are equivalent given the primitives of our model, it is of irrelevance to our analysis whether the law of large numbers that we use also holds if the alternative notion of independence were adopted. In other words, conclusions of our formal analysis are robust to the adoption of the alternative notion of independence.

\section{Concluding remarks}

Financial assets typically carry some risk idiosyncratic to them, hence, disposing incomes risk using financial assets will involve buying into the inherent idiosyncratic risk. However, standard theory argues that diversification would, in principle, reduce the inconvenience of idiosyncratic risk to arbitrarily low levels thereby making the trade-off between the two types of risk much less severe. This argument is less robust than what standard theory leads us to believe. Ambiguity aversion can actually exacerbate the tension between the two kinds of risks to the point that classes of agents may find it impossible to trade some financial assets: they can no more rely on such assets as routes for "exporting" their income risks. Thus, theoretically, the effect of ambiguity aversion on financial markets is to make the risk sharing opportunities offered by financial markets less complete than it would be otherwise. This is the principal conclusion of the exercise in this paper. This conclusion is robust, to the extent that many of the assumptions of the model presented in the last section could be substantially relaxed without losing the substance of the analytical results. First, it does not matter whether the beliefs about the economic states are ambiguous, the no-trade result still obtains. Secondly, given that diversification with replica assets doesn't work with ambiguous beliefs, one might wonder whether diversification can be achieved through assets which are not replicas (in terms of payoffs). It turns out that it does not make any difference (to the main result) if we were to relax the assumption about 'strict' replicas (see Mukerji and Tallon (1999)).

It is instructive to note the distinction between the empirical content of a theory of no-trade based on the 'lemons' problem (e.g., Morris (1997)) and the theory based on ambiguity aversion. The primitive of the former theory is asymmetric uncertainty between the transacting parties, and significantly, no trade may result even if there were no idiosyncratic component. Thus that theory, per se, does not link the presence and extent of idiosyncratic component to no trade. To obtain such a link, one has to assume, a priori, that there is sufficient asymmetric information 
only in the presence of idiosyncratic information. On the other hand, the theory based on ambiguity aversion does not require that one assumes that ambiguity is present only with idiosyncracies, or that agents have ambiguous beliefs especially with respect to payoffs of assets with idiosyncratic components. One may well begin with the primitive that ambiguity is present in a 'general' way, across all contingencies. However, since ambiguity aversion selectively attacks only those assets whose payoffs have idiosyncratic components, the link between idiosyncracy and no trade is endogenously generated in the theory based on ambiguity aversion. This positive understanding is of significance. History of financial markets is replete with episodes of increase in uncertainty leading to a thinning out of trade (or even seizing up completely) peculiarly in assets such as high yield corporate bonds ('junk' bonds) and bonds issued in "emerging markets" ( vis., Latin America, Eastern Europe and East Asia) (see Mukerji and Tallon (1999)). The understanding also explains certain institutional structures adopted in some countries to protect markets from such episodes (see Mukerji and Tallon (1999)). 


\section{Appendix A}

\section{Some formal details relating to the CEU model}

\section{Independent Product for Capacities}

We consider here the formal modeling of the idea of stochastic independence of random variables when beliefs are ambiguous. Let $y$ be a function from a given space $\tau$ to $\mathbb{R}$, and $\sigma(y)$ be the smallest $\sigma$-algebra that makes $y$ a random variable. $\tau_{n}$ denotes the $n$-fold Cartesian product of $\tau$, and $\sigma\left(y_{1}, \ldots, y_{n}\right)$ the product $\sigma$-algebra on $\tau_{n}$ generated by the $\sigma$-algebras $\left\{\sigma\left(y_{i}\right)\right\}_{i=1}^{n}$. The following definition was proposed by Gilboa and Schmeidler (1989), and earlier, by Walley and Fine (1982).

Definition 1 Let $\nu_{i}$ be a convex non-additive probability defined on $\sigma\left(y_{i}\right)$. The independent product, denoted $\bigotimes_{i=1}^{n} \nu_{i}$, is defined as follows

$$
\bigotimes_{i=1}^{n} \nu_{i}(A)=\min \left\{\left(\mu_{1} \times \ldots \times \mu_{n}\right)(A): \mu_{i} \in \mathcal{C}\left(\nu_{i}\right) \text { for } 1 \leq i \leq n\right\}
$$

for every $A \in \sigma\left(y_{1}, \ldots, y_{n}\right)$, where $\mu_{1} \times \ldots \times \mu_{n}$ is the standard additive product measure. We denote by $\otimes \nu_{i}$ any non-additive probability on $\sigma\left(y_{1}, \ldots, y_{n}, \ldots\right)$ such that for any finite class $\left\{y_{t_{1}}, \ldots, y_{t_{n}}\right\}$ it holds $\bigotimes_{i \geq 1} \nu_{i}(A)=\bigotimes_{i=1}^{n} \nu_{i}(A)$ for every $A \in \sigma\left(y_{1}, \ldots, y_{n}\right)$.

The computation of the Choquet expectation operator using product capacities is particularly simple for slice comonotonic functions (Ghirardato (1997)), defined now. Let $X_{1}, \ldots, X_{n}$ be $n$ (finite) sets and let $\Omega=X_{1} \times \ldots \times X_{n}$. Correspondingly, let $\nu_{i}$ be convex non-additive probabilities defined on algebras of subsets of $X_{i}, i=1, \ldots, n$.

Definition 2 Let $f: \Omega \rightarrow \mathbb{R}$. We say that $f$ has comonotonic $x_{i}$-sections if for every $\left(x_{1}, \ldots, x_{i-1}, x_{i+1}, \ldots, x_{n}\right),\left(x_{1}^{\prime}, \ldots, x_{i-1}^{\prime}, x_{i+1}^{\prime}, \ldots, x_{n}^{\prime}\right) \in X_{1} \times \ldots \times X_{i-1} \times X_{i+1} \times \ldots \times X_{n}$, $f\left(x_{1}, \ldots, x_{i-1}, \cdot, x_{i+1}, \ldots, x_{n}\right): X_{i} \rightarrow \mathbb{R}$, and $f\left(x_{1}^{\prime}, \ldots, x_{i-1}^{\prime}, \cdot, x_{i+1}^{\prime}, \ldots, x_{n}^{\prime}\right): X_{i} \rightarrow \mathbb{R}$ are comonotonic functions. $f$ is called slice-comonotonic if it has comonotonic $x_{i}$-sections for every $i \in\{1, \ldots, n\}$.

The following fact follows from Proposition 7 and Theorem 1 in Ghirardato (1997).

Fact 1 Suppose that $f: \Omega \rightarrow \mathbb{R}$ is slice comonotonic. Then

$$
\mathbb{C E}_{\otimes \nu_{i}} f\left(x_{1}, \ldots, x_{n}\right)=\mathbb{C E}_{\nu_{1}} \ldots \mathbb{C E}_{\nu_{n}} f\left(x_{1}, \ldots, x_{n}\right)
$$

In what follows we verify that Fact 1 applies to the calculation of Choquet expected utility of an agent's contingent consumption vector. As in the main text $\Omega=S \times\{0,1\}^{n}$ be the state space, with generic element $\omega=\left(s, t_{1}, \ldots, t_{n}\right)=(s, t)$. For a given $h$ let $x(\omega)=x_{h, n}^{s, t}, h$ 's consumption at state $\omega=(s, t)$. Finally let $u: \mathbb{R} \rightarrow \mathbb{R}$ denote the strictly increasing utility index. It will be shown that composite function, $u \circ x():. \Omega \rightarrow \mathbb{R}$ is slice comonotonic, and therefore, the calculation of $\mathbb{C E} u(x(\omega))$ may obtain as in Fact 1. Recall,

$$
x(\omega)=x(s, t)=e_{h}^{s}+b_{h}+\widetilde{z}_{h}\left(\sum_{i=1}^{n} \frac{y^{s}+y\left(t_{i}\right)}{n}\right)
$$


where $\widetilde{z}_{h}$ is the holding of the diversified portfolio consisting of $1 / n$ units of each financial asset. We first show that $x($.$) is slice-comonotonic. This is done by demonstrating, in turn, that x$ has comonotonic $s$-sections and comonotonic $t_{j}$-sections.

Fix $t=\left(t_{1}, \ldots, t_{n}\right)$ and $t^{\prime}=\left(t_{1}^{\prime}, \ldots, t_{n}^{\prime}\right)$. Assume that $x(s, t) \geq x\left(s^{\prime}, t\right)$. Then, as required in definition 2 (slice comonotonicity), we want to show that $x(s, t) \geq x\left(s^{\prime}, t\right)$. Now,

$$
\begin{aligned}
x(s, t) & \geq x\left(s^{\prime}, t\right) \\
& \Leftrightarrow e_{h}^{s}+b_{h}+\widetilde{z}_{h}\left(\sum_{i=1}^{n} \frac{y^{s}+y\left(t_{i}\right)}{n}\right) \geq e_{h}^{s^{\prime}}+b_{h}+\widetilde{z}_{h}\left(\sum_{i=1}^{n} \frac{y^{s^{\prime}}+y\left(t_{i}\right)}{n}\right) \\
& \Leftrightarrow e_{h}^{s}+b_{h}+\widetilde{z}_{h} y^{s} \geq e_{h}^{s^{\prime}}+b_{h}+\widetilde{z}_{h} y^{s^{\prime}} \\
& \Leftrightarrow e_{h}^{s}+b_{h}+\widetilde{z}_{h}\left(\sum_{i=1}^{n} \frac{y^{s}+y\left(t_{i}^{\prime}\right)}{n}\right) \geq e_{h}^{s^{\prime}}+b_{h}+\widetilde{z}_{h}\left(\sum_{i=1}^{n} \frac{y^{s^{\prime}}+y\left(t_{i}^{\prime}\right)}{n}\right) \\
& \Leftrightarrow x\left(s, t^{\prime}\right) \geq x\left(s^{\prime}, t^{\prime}\right)
\end{aligned}
$$

Hence, $x$ has comonotonic $s$-sections.

Next, fix $\left(s, t_{-j}\right)$ where $t_{-j}=\left(t_{1}, \ldots t_{j-1}, t_{j+1}, \ldots, t_{n}\right)$ and $\left(s^{\prime}, t_{-j}^{\prime}\right)$. Now,

$$
\begin{aligned}
x\left(s, t_{-j}, t_{j}\right) & \geq x\left(s, t_{-j}, t_{j}^{\prime}\right) \\
& \Leftrightarrow e_{h}^{s}+b_{h}+\widetilde{z}_{h}\left(\sum_{i \neq j} \frac{y^{s}+y\left(t_{i}\right)}{n}+\frac{\left(y^{s}+y\left(t_{j}\right)\right)}{n}\right) \\
& \geq e_{h}^{s}+b_{h}+\widetilde{z}_{h}\left(\sum_{i \neq j} \frac{y^{s}+y\left(t_{i}\right)}{n}+\frac{\left(y^{s}+y\left(t_{j}^{\prime}\right)\right)}{n}\right) \\
& \Leftrightarrow y\left(t_{j}\right) \geq y\left(t_{j}^{\prime}\right) \\
& \Leftrightarrow x\left(s^{\prime}, t_{-j}^{\prime}, t_{j}\right) \geq x\left(s^{\prime}, t_{-j}^{\prime}, t_{j}^{\prime}\right)
\end{aligned}
$$

Repeating this, one shows that $x$ has comonotonic $t_{j}$-sections, for all $j=1, \ldots, n$. Hence, $x$ is slice comonotonic.

Now, it is possible see that slice comonotonicity of $u \circ x():. \Omega \rightarrow \mathbb{R}$ follows readily from the assumption that $u$ is strictly increasing. To this end, notice:

$$
x(s, t) \geq x\left(s^{\prime}, t\right) \Leftrightarrow u(x(s, t)) \geq u\left(x\left(s^{\prime}, t\right)\right)
$$

and

$$
x\left(s, t_{-j}, t_{j}\right) \geq x\left(s, t_{-j}, t_{j}^{\prime}\right) \Leftrightarrow u\left(x\left(s, t_{-j}, t_{j}\right)\right) \geq u\left(x\left(s, t_{-j}, t_{j}^{\prime}\right)\right)
$$

Law of Large Numbers for Capacities: (Marinacci (1996) Theorem 7.7, Walley and Fine (1982))

Let $y$ be a function from a given (countably) finite space $\Omega$ to the real line $\mathbb{R}$, and $\sigma(y)$ the smallest $\sigma$-algebra that makes $y$ a random variable. $\Omega^{n}$ denotes the $n$-fold Cartesian product of $\Omega$, and $\sigma\left(y_{1}, \ldots, y_{n}\right)$ the product $\sigma$-algebra on $\Omega^{n}$ generated by the $\sigma$-algebras $\left\{\sigma\left(y_{i}\right)\right\}_{i=1}^{n}$. Set $S_{n}=\frac{1}{n} \sum_{i=1}^{n} y_{i}$. Let each $\nu_{i}$ be a convex capacity on $\sigma\left(y_{i}\right)$, and let $\left\{y_{i}\right\}_{i \geq 1}$ be a sequence of random variables independent and identically distributed relative to $\otimes \nu_{i}$. Set $S_{n}=\frac{1}{n} \sum_{i=1}^{n} y_{i}$. Suppose both $\mathbb{C E}_{\nu_{1}}\left(y_{1}\right)$ and $\mathbb{C E}_{\nu_{1}}\left(-y_{1}\right)$ exist. Then 
1. $\otimes \nu_{i}\left(\left\{\begin{aligned} \omega \in \Omega^{\infty}: \mathbb{C E}_{\nu_{1}}\left(y_{1}\right) & \leq \liminf _{n} S_{n}(\omega) \leq \limsup \sup _{n} S_{n}(\omega) \\ & \leq-\mathbb{C E}_{\nu_{1}}\left(-y_{1}\right)\end{aligned}\right\}\right)=1$

2. $\otimes \nu_{i}\left(\left\{\begin{array}{c}\omega \in \Omega^{\infty}: \mathbb{C E}_{\nu_{1}}\left(y_{1}\right)<\liminf _{n} S_{n}(\omega) \leq \limsup S_{n} S_{n}(\omega) \\ <-\mathbb{C E}_{\nu_{1}}\left(-X_{1}\right)\end{array}\right\}\right)=0$.

3. $\otimes \nu_{i}\left(\left\{\omega \in \Omega^{\infty}: \mathbb{C E}_{\nu_{1}}\left(y_{1}\right) \neq \liminf _{n} S_{n}(\omega)\right\}\right)=0$.

4. $\otimes \nu_{i}\left(\left\{\omega \in \Omega^{\infty}:-\mathbb{C E}_{\nu_{1}}\left(-y_{1}\right) \neq \lim \sup _{n} S_{n}(\omega)\right\}\right)=0$.

\section{Appendix B}

\section{Proofs of results in the main text}

Proof of the lemma: Suppose w.l.o.g. $q^{z^{i}} \geq q^{z^{i^{\prime}}}$ for some $i, i^{\prime} \in\{1, \ldots, n\}$. First we show that $z_{h, n}^{i} \leq z_{h, n}^{i^{\prime}}, \forall h \in\{1, \ldots, H\}$. Indeed, assume $z_{h, n}^{i}>z_{h, n}^{i^{\prime}}$ for some and construct the portfolio $\bar{z}_{h, n}$ as follows:

$$
\bar{z}_{h, n}^{i}=z_{h, n}^{i}-\varepsilon, \bar{z}_{h, n}^{i^{\prime}}=z_{h, n}^{i^{\prime}}+\frac{q^{z^{i}}}{q^{z^{i^{\prime}}}} \varepsilon, \text { and } \bar{z}_{h, n}^{j}=z_{h, n}^{j} \forall j \neq i, i^{\prime} .
$$

where $\varepsilon$ is small enough so that $\bar{z}_{h, n}^{i}>\bar{z}_{h, n}^{i^{\prime}}$. Note, $\bar{z}_{h, n}$ is budget feasible. Let

$$
\bar{x}_{h, n}^{s, t} \equiv e_{h}^{s}+b_{h, n}+\sum_{i=1}^{n} z_{h, n}^{i}\left(y^{s}+y\left(t_{i}\right)\right) \text { for } s=1,2
$$

Because $x_{h, n}^{s, t}$ and $\bar{x}_{h, n}^{s, t}$ are comonotonic, and $u_{h}$ is strictly increasing, it follows from Definition 1 that there exists an additive product measure $\mu$, where $\mu \equiv \times_{i=1}^{n} \mu_{i}$, and $\mu_{i}: 2^{\{0,1\}} \rightarrow[0,1]$ are additive measures, such that,

$$
\begin{aligned}
\mathbb{C E}_{\pi \otimes \nu}\left(x_{h, n}^{s, t}\right) & =\mathbb{E}_{\pi \times \mu}\left(x_{h, n}^{s, t}\right), \mathbb{C E}_{\pi \otimes \nu}\left(\bar{x}_{h, n}^{s, t}\right)=\mathbb{E}_{\pi \times \mu}\left(\bar{x}_{h, n}^{s, t}\right), \text { and }, \\
\mathbb{C E}_{\pi \otimes \nu}\left(u_{h}\left(x_{h, n}^{s, t}\right)\right) & =\mathbb{E}_{\pi \times \mu}\left(u_{h}\left(x_{h, n}^{s, t}\right)\right) \\
\mathbb{C E}_{\pi \otimes \nu}\left(u_{h}\left(\bar{x}_{h, n}^{s, t}\right)\right) & =\mathbb{E}_{\pi \times \mu}\left(u_{h}\left(\bar{x}_{h, n}^{s, t}\right)\right), s=1,2, \forall t \in \tau_{n} .
\end{aligned}
$$

Furthermore, $\mathbb{E}_{\mu}\left(x_{h, n}^{s, t} \mid s\right)=\mathbb{E}_{\mu}\left(\bar{x}_{h, n}^{s, t} \mid s\right)+\mathbb{E}_{\mu_{i}} \varepsilon y\left(t_{i}\right)-\mathbb{E}_{\mu_{i^{\prime}}} \varepsilon y\left(t_{i^{\prime}}\right), s=1,2$. Next, notice

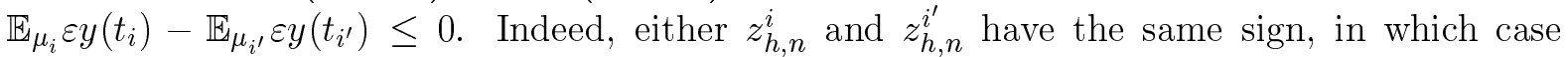
$\mu_{i}=\mu_{i^{\prime}}$ and $\mathbb{E}_{\mu_{i}} \varepsilon y\left(t_{i}\right)-\mathbb{E}_{\mu_{i^{\prime}}} \varepsilon y\left(t_{i^{\prime}}\right)=0$. Or $z_{h, n}^{i}>0>z_{h, n}^{i^{\prime}}$ and then

$$
\left.\mathbb{E}_{\mu_{i}} \varepsilon y\left(t_{i}\right)-\mathbb{E}_{\mu_{i^{\prime}}} \varepsilon y\left(t_{i^{\prime}}\right)\right)=\varepsilon\left[1-\nu^{0}-\nu^{1}\right][y(0)-y(1)] \leq 0
$$

Hence, $\bar{x}^{s}$ stochastically dominates $x^{s}$. Given $u^{\prime \prime}<0$, therefore, $\mathbb{E}_{\pi \times \mu} u_{h}\left(\bar{x}_{h, n}^{s, t}\right)>\mathbb{E}_{\pi \times \mu} u_{h}\left(x_{h, n}^{s, t}\right)$. As a consequence, $\mathbb{C E}_{\pi \otimes \nu} u_{h}\left(\bar{x}_{h, n}^{s, t}\right)>\mathbb{C E}_{\pi \otimes \nu} u_{h}\left(x_{h, n}^{s, t}\right)$. But this is a contradiction to the hypothesis that $\left(\mathbf{q}_{n},\left(\mathbf{b}_{n}, \mathbf{z}_{n}, \mathbf{x}_{n}\right)\right)$ is an equilibrium. $\therefore z_{h, n}^{i} \leq z_{h, n}^{i^{\prime}}, \forall h \in\{1, \ldots, H\}$.

Since, $\left(\mathbf{q}_{n},\left(\mathbf{b}_{n}, \mathbf{z}_{n}, \mathbf{x}_{n}\right)\right)$ is an equilibrium, $\sum_{h=1}^{H} z_{h, n}^{i}=\sum_{h=1}^{H} z_{h, n}^{i^{\prime}}=0$. Therefore, using the fact that $z_{h, n}^{i} \leq z_{h, n}^{i^{\prime}}$ for all $h$, we get that $z_{h, n}^{i}=z_{h, n}^{i^{\prime}}$ for all $h$.

Proof of the Theorem: The maximization problem $\widetilde{\mathcal{P}}_{h \infty}$ given asset prices $\widetilde{\mathbf{q}}_{\infty}$ may be written as follows:

$$
\begin{gathered}
\max \mathbb{E}_{\pi \otimes \nu} u_{h}\left(e_{h}^{s}+b_{h, \infty}+\tilde{z}_{h, \infty}\left[\lim _{n \rightarrow \infty} \sum_{i=1}^{n} \frac{y^{s}+y\left(t_{i}\right)}{n}\right]\right) \\
\text { s.t. } b_{h, \infty}+\tilde{q}_{\infty} \tilde{z}_{h, \infty}=0
\end{gathered}
$$


And the maximization problem $\mathcal{P}_{h}$, solved by the agent in an economy without idiosyncracy, given asset prices $\mathbf{q}=\widetilde{\mathbf{q}}_{\infty}$ :

$$
\begin{gathered}
\max \sum_{s \in\{1,2\}} \pi(s) u_{h}\left(e_{h}^{s}+b_{h, n}+z_{h} \bar{y}^{s}\right) \\
\text { s.t. } b_{h}+\tilde{q}_{\infty} z_{h}=0
\end{gathered}
$$

If $n \rightarrow \infty$, by the law of large numbers, with probability 1 an unit of the portfolio $\tilde{z}_{n}$ yields a payoff of $y^{s}+\mathbb{E}_{t \in\{0,1\}} y(t) \equiv \bar{y}^{s}$ units. That is, $\lim _{n \rightarrow \infty} \sum_{i=1}^{n}\left(\frac{\left(y^{s}+y\left(t_{i}\right)\right)}{n}\right) \stackrel{a . s}{\longrightarrow} \bar{y}^{s}$. Recall, the financial asset $z$ yields $\bar{y}^{s}$ units of the good in the economic states $s=1,2$.

Hence, $\left(\mathbf{b}_{\infty}, \widetilde{\mathbf{z}}_{\infty}\right)$ solves the maximization problem $\widetilde{\mathcal{P}}_{h \infty}$ at prices $\left(\widetilde{\mathbf{q}}_{\infty}\right)$, if and only if $\left(\mathbf{b}_{\infty}, \widetilde{\mathbf{z}}_{\infty}\right)$ also solves the maximization problem $\mathcal{P}_{h}$ at prices $\left(\widetilde{\mathbf{q}}_{\infty}\right)$.

Finally note, if $\left(\widetilde{\mathbf{q}}_{\infty},\left(\mathbf{b}_{\infty}, \widetilde{\mathbf{z}}_{\infty}, \mathbf{x}_{\infty}\right)\right)$ describes an equilibrium of the $n$-financial assets economy with idiosyncracy it must be that $\left(\mathbf{b}_{\infty}, \widetilde{\mathbf{z}}_{\infty}\right)$ satisfies the conditions of (asset) market clearing at the price vector $\widetilde{\mathbf{q}}_{\infty}$. Hence, $\left(\widetilde{\mathbf{q}}_{\infty}\right)$ will also clear asset markets in the economy without idiosyncracy. Conversely, if $\left(\widetilde{\mathbf{q}}_{\infty},\left(\mathbf{b}_{\infty}, \widetilde{\mathbf{z}}_{\infty}, \mathbf{x}_{\infty}\right)\right)$ describes an equilibrium of the economy without idiosyncracy then $\left(\widetilde{\mathbf{q}}_{\infty}\right)$ will also clear asset markets in the $n$-financial assets economy with idiosyncracy.

Proof of the Main Theorem: Consider $\widetilde{\mathcal{P}}_{h n}$, the maximization problem in the $n$-financial asset economy with idiosyncracy. Suppose that, at equilibrium there exists $h^{\prime}$ such that $\tilde{z}_{h^{\prime}, n} \neq 0$, say $\tilde{z}_{h^{\prime}, n}>0$. Then, there must be $h^{\prime \prime}$ such that $\tilde{z}_{h^{\prime \prime}, n}<0$. Next, since $\tilde{z}_{h^{\prime}, n}>0$, and $y(0)<y(1)$, Fact 1 together with the fact that $u_{h}\left(x_{h, n}^{\omega}\right)$ is slice-comonotonic (see Appendix A), implies that $\mathbb{C} \mathbb{E}_{\pi \otimes \nu} u_{h^{\prime}}\left(x_{h^{\prime}, n}^{s, t}\right)$ is a standard expectation with respect to the additive measure $\pi \times \underline{\mu}(t)$, where $\underline{\mu}(t)=\left(1-\nu^{1}\right)^{n_{0}} \times\left(\nu^{1}\right)^{n-n_{0}}, n_{0}$ being the number of financial assets whose idiosyncratic payoff is $y(0)$ at state $(s, t)$. This is because $x_{h^{\prime}, n}^{s,\left(t_{i}, t_{-i}\right)}$ is necessarily smaller at a state $\left(s,\left(0, t_{-i}\right)\right)$ than at the state $\left(s,\left(1, t_{-i}\right)\right), s=1,2$. The first order conditions of the problem $\widetilde{\mathcal{P}}_{h^{\prime} n}$ (for agent $h^{\prime}$ ) then give:

$$
\tilde{q}_{n}=\frac{\mathbb{E}_{\pi \times \underline{\mu}}\left[\sum_{i=1}^{n}\left\{\left(\frac{y^{s}+y\left(t_{i}\right)}{n}\right) u_{h^{\prime}}^{\prime}\left(x_{h^{\prime}, n}^{s, t}\right)\right\}\right]}{\mathbb{E}_{\pi \times \underline{\mu}}\left[u_{h^{\prime}}^{\prime}\left(x_{h^{\prime}, n}^{s, t_{i}}\right)\right]}
$$

Notice, for $s=1,2, x_{h^{\prime}, n}^{s, t}$ and $\sum_{i=1}^{n}\left(\frac{y^{s}+y\left(t_{i}\right)}{n}\right)$ are positively dependent given $s$ (see Magill and Quinzii (1996)) since $\tilde{z}_{h^{\prime}, n}>0$. Hence, because $u^{\prime \prime}(\cdot)<0$,

$$
\text { Covariance }\left(\sum_{i=1}^{n}\left(\frac{y^{s}+y\left(t_{i}\right)}{n}\right), u_{h^{\prime}}^{\prime}\left(x_{h^{\prime}, n}^{s, t}\right)\right)<0, \text { given } s \text {. }
$$

Now,

$$
\begin{aligned}
\mathbb{E}_{\underline{\mu}}\left[\sum_{i=1}^{n}\left\{\left(\frac{y^{s}+y\left(t_{i}\right)}{n}\right) u_{h^{\prime}}^{\prime}\left(x_{h^{\prime}, n}^{s, t}\right)\right\}\right]= & \text { Covariance }\left(\sum_{i=1}^{n}\left(\frac{y^{s}+y\left(t_{i}\right)}{n}\right), u_{h^{\prime}}^{\prime}\left(x_{h^{\prime}, n}^{s, t}\right)\right) \\
& +\mathbb{E}_{\underline{\mu}}\left[\sum_{i=1}^{n}\left(\frac{y^{s}+y\left(t_{i}\right)}{n}\right)\right] \mathbb{E}_{\underline{\mu}} u_{h^{\prime}}^{\prime}\left(x_{h^{\prime}, n}^{s, t}\right) .
\end{aligned}
$$

Thus,

$$
\mathbb{E}_{\underline{\mu}}\left[\sum_{i=1}^{n}\left\{\left(\frac{y^{s}+y\left(t_{i}\right)}{n}\right) u_{h^{\prime}}^{\prime}\left(x_{h^{\prime}, n}^{s, t}\right)\right\}\right]<\mathbb{E}_{\underline{\mu}}\left[\sum_{i=1}^{n}\left(\frac{y^{s}+y\left(t_{i}\right)}{n}\right)\right] \mathbb{E}_{\underline{\mu}} u_{h^{\prime}}^{\prime}\left(x_{h^{\prime}, n}^{s, t}\right) .
$$


Hence,

$$
\begin{aligned}
\tilde{q}_{n}<\frac{\sum_{s=1}^{2} \pi(s) \mathbb{E}_{\underline{\mu}}\left[\sum_{i=1}^{n}\left(\frac{y^{s}+y\left(t_{i}\right)}{n}\right)\right] \mathbb{E}_{\underline{\mu}} u_{h^{\prime}}^{\prime}\left(x_{h^{\prime}, n}^{s, t}\right)}{\sum_{s=1}^{2} \pi(s) \mathbb{E}_{\underline{\mu}}\left[u_{h^{\prime}}^{\prime}\left(x_{h^{\prime}, n}^{s, t}\right)\right]} \\
\Rightarrow \tilde{q}_{n}<\max _{s}\left\{\mathbb{E}_{\underline{\mu}}\left[\sum_{i=1}^{n}\left(\frac{y^{s}+y\left(t_{i}\right)}{n}\right)\right]\right\} \\
\Rightarrow \tilde{q}_{n}<y^{\bar{s}}+\left(1-\nu^{1}\right) y(0)+\nu^{1} y(1)
\end{aligned}
$$

Consider next, $h^{\prime \prime}$ such that $\tilde{z}_{h^{\prime \prime}, n}<0$. By a reasoning similar to that followed for the agent $h^{\prime}$ (noticing $x_{h^{\prime \prime}, n}^{s, t}$ and $\sum_{i=1}^{n}\left(\frac{\left(y^{s}+y\left(t_{i}\right)\right)}{n}\right)$ are negatively dependent given $s$ ) one gets

$$
\tilde{q}_{n}>y^{\underline{s}}+\left(1-\nu^{0}\right) y(1)+\nu^{0} y(0) .
$$

Therefore, a necessary condition for having an equilibrium with $\tilde{z}_{h, n} \neq 0$ for at least some $h$ is that $y^{\bar{s}}-y^{\underline{s}}>\left(1-\nu^{0}-\nu^{1}\right)(y(1)-y(0))$. Set $\overline{\mathcal{A}}=\frac{y^{\bar{s}}-y^{\underline{\underline{s}}}}{y(1)-y(0)} \in(0,1)$. If $1-\nu^{0}-\nu^{1}>\overline{\mathcal{A}}$, then $\tilde{z}_{h, n}=0$ for all $h$, at any equilibrium.

Finally, note if $n \rightarrow \infty, \mathbb{C E}_{\pi \otimes \nu} u_{h^{\prime}}\left(x_{h^{\prime}, n}^{s, t}\right)$ is just a standard expectation operator with respect to the additive measure $\pi \times \underline{\mu}(t)$, where $\underline{\mu}(t)$ is such that

$$
\underline{\mu}\left(\left\{t: \lim _{n \rightarrow \infty} \sum_{i=1}^{n}\left(\frac{\left(y^{s}+y\left(t_{i}\right)\right)}{n}\right)=y^{s}+\left(1-\nu^{1}\right) y(0)+\nu^{1} y(1)\right\}\right)=1 .
$$

The proof then proceeds as in the case of finite $n$ except that the inequality (1) reads

$$
\lim _{n \rightarrow \infty} \tilde{q}_{n} \leq y^{\bar{s}}+\left(1-\nu^{1}\right) y(0)+\nu^{1} y(1)
$$

and the inequality (2) reads $\lim _{n \rightarrow \infty} \tilde{q}_{n} \geq y^{\underline{s}}+\left(1-\nu^{0}\right) y(1)+\nu^{0} y(0)$. 


\section{References}

Bewley, T. (1986): "Knightian Decision Theory: Part," Discussion Paper 807, Cowles Foundation.

Carroll, C. (1992): "The Buffer Stock Theory of Savings ; some Macroeconomic Evidence," Brookings Papers on Economic Activity, 2, 61-135.

Chateauneuf, A., R. Dana, and J.-M. Tallon (2000): "Optimal Risk Sharing Rules and Equilibria with Choquet-Expected-Utility," Journal of Mathematical Economics, 34, 191-214.

Deaton, A., And C. Paxson (1994): "Intertemporal Choice and Inequality," Journal of Political Economy, 102(3), 437-467.

Dow, J., and S. Werlang (1992): "Uncertainty Aversion, Risk Aversion, and the Optimal Choice of Portfolio," Econometrica, 60(1), 197-204.

Epstein, L. (1999): "A Definition of Uncertainty Aversion," Review of Economic Studies, 66, 579-608.

Epstein, L., And T. WAng (1994): "Intertemporal Asset Pricing under Knightian Uncertainty," Econometrica, 62(3), 283-322.

Fishburn, P. (1993): "The Axioms and Algebra of Ambiguity," Theory and Decision, 34, 119-137.

Ghirardato, P. (1997): "On Independence for Non-Additive Measures, with a Fubini Theorem," Journal of Economic Theory, 73, 261-291.

Ghirardato, P., And M. Marinacci (1997): "Ambiguity Aversion Made Precise: A Comparative Foundation and some Implications," Social science w.p. 1026, CalTech.

Gilboa, I., And D. Schmeidler (1989): "Maxmin Expected Utility with a Non-Unique Prior," Journal of Mathematical Economics, 18, 141-153.

Hayashi, F., J. Altonji, and L. Kotlikof (1996): "Risk Sharing Between and Within Families," Econometrica, 64(2), 261-294.

Hendon, E., H. Jacobsen, B. Sloth, and T. Tranaes (1996): "The Product of Capacities and Belief Functions," Mathematical Social Sciences, 32(2), 95-108.

Kelsey, D., And F. Milne (1995): "The Arbitrage Pricing Theorem with Non-Expected Utility Preferences," Journal of Economic Theory, 65(2), 557-574.

LucAs, R. (1978): “Asset Prices in an Exchange Economy," Econometrica, 46, 1429-1445.

Mace, B. (1991): "Full Insurance in the Presence of Aggregate Uncertainty," Journal of Political Economy, 99(5), 928-956.

Magill, M., ANd M. QUinziI (1996): Theory of Incomplete Markets, vol. 1. MIT Press.

MarinaCCI, M. (1996): "Limit Laws for Non-Additive Probabilities, and their Frequentist Interpretation," mimeo.

MARINACCI, M. (1999): "Limit Laws for Non-Additive Probabilities and Their Frequentist Interpretation," Journal of Economic Theory, 84, 145-195.

Morris, S. (1997): "Risk, Uncertainty and Hidden Information," Theory and Decision, 42(3), 235-269. 
MukerJI, S. (1997): "Understanding the Nonadditive Probability Decision Model," Economic Theory, 9(1), 23-46.

MukerJi, S. (1998): "Ambiguity Aversion and Incompleteness of Contractual Form," American Economic Review, 88(5), 1207-1231.

MukerJI, S., AND J.-M. TAllon (1999): "Ambiguity Aversion and Incompleteness of Financial Markets-Extended Version," Mimeo. 99-28, Cahiers de la Maison des Sciences Economiques, Universite Paris I, available for download at http://panoramix.univparis1.fr/EUREQUA/annuaire/tallon/tallon.htm.

SchmeIdler, D. (1989): "Subjective Probability and Expected Utility Without Additivity," Econometrica, 57(3), 571-587.

Walley, P., And L. Fine (1982): "Towards a Frequentist Theory of Upper and Lower Probability," Annals of Statistics, 10, 741-761.

Werner, J. (1997): "Diversification and Equilibrium in Securities Markets," Journal of Economic Theory, 75, 89-103.

Zeldes, S. (1989): "Consumption and Liquidity: An Empirical Investigation," Journal of Political Economy, 97, 305-346. 